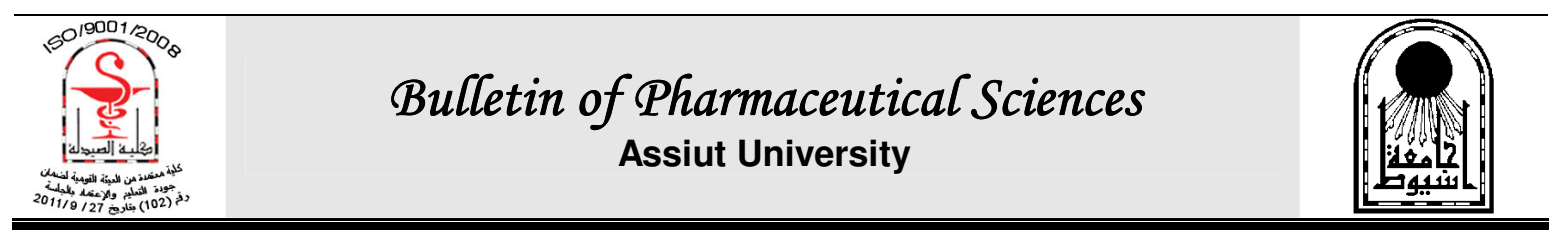

\title{
SYNTHESIS OF NOVEL PYRAZOLE DERIVATIVES BEARING 1,2,4- TRIAZOLE MOIETY AS POTENTIAL ANTICANCER AGENTS
}

\author{
Mostafa H. Abdelrahman ${ }^{1 *}$ and Mamdouh M. $\mathrm{Ali}^{2}$ \\ ${ }^{1}$ Department of Organic Chemistry, Faculty of Pharmacy, Al-Azhar University, Assiut 71524, \\ Egypt \\ ${ }^{2}$ Department of Biochemistry, Division of Genetic Engineering and Biotechnology, National \\ Research Centre, Dokki 12622, Giza, Egypt
}

\begin{abstract}
A series of novel 3-(1H-pyrazol-3-yl)-4H-1,2,4-triazole derivatives were synthesized and the structure of the prepared compounds was fully characterized by ${ }^{1} \mathrm{H} N M R,{ }^{13} \mathrm{C} \mathrm{NMR}$, and HRESI-MS. Six of the prepared compounds were screened for in-vitro cytotoxicity against different cancer cell lines at National Cancer Institute (NCI), USA. Compound 7e exhibited a broad-spectrum of anticancer activity against different cancer cell lines without pronounced selectivity. Moreover, the anticancer activity of the prepared compounds was also evaluated against different human cancer cell lines including breast MCF-7, lung A549 as well as the human normal melanocyte (HFB4) using doxorubicin as a reference drug. Compounds $7 l$ and 7 o exhibited remarkable anticancer activity similar to or more potent than doxorubicin against breast MCF-7 and Lung A549 cell lines.
\end{abstract}

\section{INTRODUCTION}

Cancer is one of the leading causes of morbidity and mortality worldwide ${ }^{1}$. Treatment of cancer is associated with various side effects which include bone marrow, depression, alopecia, and hepatotoxicity. In addition, the development of resistance against the existing anticancer drugs and cytotoxicity of anticancer drugs to the normal cells are other major problems in cancer therapy so the development of new anticancer therapeutic agents with improved efficacy and minimal side effects is one of the fundamental goals in medicinal chemistry.

Several pyrazole derivatives have been reported to possess diverse pharmacological activities such as anti-inflammatory ${ }^{2-4}$, analgesic $^{5}, \quad$ anticancer $^{6-12}$, antiviral ${ }^{13}$, antimicrobial $^{14 \& 15}$, antitubercular ${ }^{16 \& 17}$, antihyperglycemic ${ }^{18}, \quad$ antidepressant $^{19}$, anticonvulsant ${ }^{20 \& 21}$, and antihepatotoxic ${ }^{22}$. Additionally, the pyrazole ring is a prominent structural motif found in numerous pharmaceutically active compounds such as selective COX-2 inhibitor (Celecoxib) ${ }^{23}$, nonsteroidal anti-inflammatory drug (Lonazolac) ${ }^{24}$, phosphodiesterase inhibitor (Sildenafil) ${ }^{25}$, clinically approved anticancer agents (Ruxolitinib) ${ }^{26}$ and antiobesity cannabinoid drug (Rimonabant) ${ }^{27}$ (Fig. 1). A cannabinoid based medicine is already licensed for treatment of the nausea and vomiting associated with chemotherapy in cancer patients $^{28}$. Cannabinoids possess antineoplastic effect against types of growth, such as thyroid epithelioma, skin carcinomas, uterine carcinoma, breast cancer, prostate carcinoma, and neuroblastoma ${ }^{29}$. Cannabinoids might offer a relatively comprehensive medical treatment for cancer patients as they could inhibit tumour cell proliferation, induce appetite, and reduce inflammation and pain.

Meanwhile, 1,2,4-triazoles are an important class of heterocyclic compounds with a wide range of biological activities such as antimicrobial ${ }^{30 \& 31}$, antitubercular ${ }^{32}$, anticancer $^{33-38}$, antiviral ${ }^{39}$, anticonvulsant ${ }^{40}$,

Received in 16/2/2016 \& Accepted in 3/4/2016 


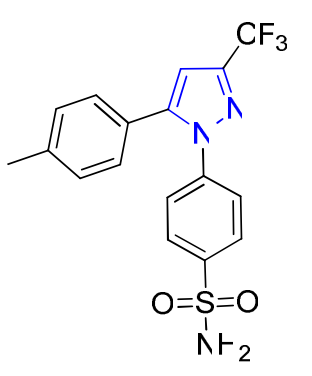

Celecoxib

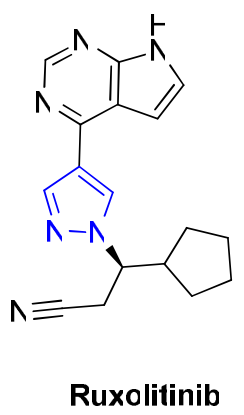

Ruxolitinib

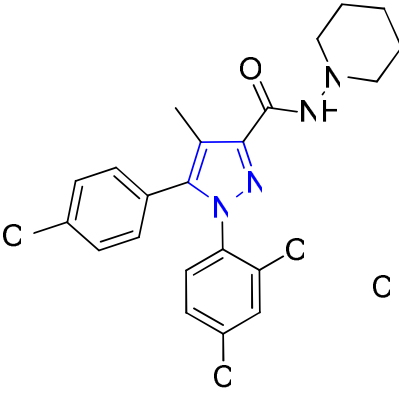

Rimonabant

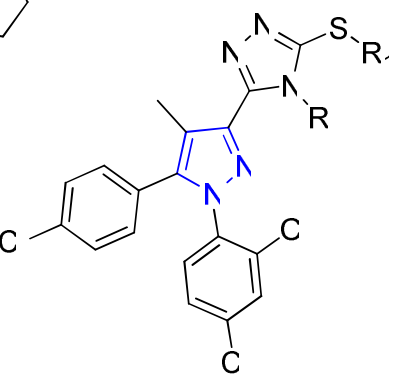

Frepared compounds

Fig. 1: Structures of some pharmaceutically active compounds containing pyrazole moiety and general structure of the prepared 3-(1H-pyrazol-3-yl)-4H-1,2,4-triazoles.

antiinflammatory $^{41}$, analgesic ${ }^{42}$, antihypertensive $^{43}$, and antidepressant ${ }^{44}$. Furthermore, the 1,2,4-triazole nucleus has been incorporated into a variety of therapeutically interesting drug candidates including antifungal (Fluconazole), antiviral (Ribavarin), antimigraine (Rizatriptan), and antianxiety compounds (Alprazolam) ${ }^{36 \& 45}$.

Based on the above mentioned studies, the present work gathers the two bioactive entities, 1,5-diarylpyrazole functionality which the main nucleus in Rimonabant and the 1,2,4-triazole moiety in one compact structure for the purpose of synergistic effect. The synthesis and in-vitro anticancer activities of the prepared 3(1H-pyrazol-3-yl)-4H-1,2,4-triazole derivatives were reported here.

\section{MATERIALS AND METHODS}

All the chemicals used were of analytical grade and purified by standard methods prior to use. Silica gel column chromatography was carried out using kieselgel 60 (merck). TLC analysis was performed on aluminium-backed plates coated with silica gel $60 \mathrm{~F}_{254}$ (Merck). Melting points were determined using a Gallen Kamp melting point apparatus and are uncorrected. Components were visualized using potassium permanganate solution and UV light. NMR Spectra were taken using a Varian Unity INOVA $400 \mathrm{MHz}$ spectrometer for proton and $101 \mathrm{MHz}$ for carbon at university of Aberdeen. All numbers referring to NMR data obtained are in parts per million (ppm). High resolution mass spectrometric data were obtained using Thermo Instruments MS system (LTQ XL/LTQ Orbitrap Discovery) coupled to a Thermo Instruments HPLC system (Accela PDA detector, Accela PDA autosampler and Pump) at university of Aberdeen.

\section{Chemistry}

Synthesis of lithium Salt of 4-(4chlorophenyl)-3-methyl-2,4-dioxobutyric acid ethyl ester (2)

To a magnetically stirred solution of lithium bis(trimethylsilyl)amide (LHMDS) (32 $\mathrm{mL}, 1.0 \mathrm{M}$ in THF, $32 \mathrm{mmol}$ ) in diethyl ether $(80 \mathrm{~mL})$ at $-78{ }^{\circ} \mathrm{C}$ a solution of 4chloropropiophenone $\mathbf{1}$ (4 $\mathrm{g}, 24 \mathrm{mmol}$ ) in diethyl ether $(20 \mathrm{~mL})$ was added in a drop wise manner under nitrogen atmosphere. After the mixture was stirred at the ambient temperature for period of $1 \mathrm{~h}$, diethyl oxalate ( $4 \mathrm{~g}, 27.78$ mmol) was added in a drop wise manner. The reaction mixture was allowed to warm to room temperature and stirred for another $16 \mathrm{~h}$. The formed precipitate was collected by filtration, washed with diethyl ether, and dried under vacuum to afford the crude lithium salt $\mathbf{2}$ as a yellowish solid.

Synthesis of ethyl 5-(4-chlorophenyl)-1-(2,4dichlorophenyl)-4-methyl-1H-pyrazole-3carboxylate (3)

To a solution of the lithium salt 2 ( $5 \mathrm{~g}$, $18.18 \mathrm{mmol})$ in ethanol $(50 \mathrm{~mL}) \quad 2,4-$ dichlorophenylhydrazine hydrochloride (4.67 $\mathrm{g}, 21.82 \mathrm{mmol}$ ) was added at room temperature. The resulting mixture was stirred for additionally $20 \mathrm{~h}$. The formed precipitate 
was collected by filtration, washed with ethanol and $\mathrm{Et}_{2} \mathrm{O}$, and dried under vacuum to give a light-yellow solid of the hydrazone. The solid was dissolved in acetic acid $(30 \mathrm{~mL})$ and heated to reflux for $24 \mathrm{~h}$. The reaction mixture was poured into ice water, and extracted with ethyl acetate $(3 \times 40 \mathrm{~mL})$. The EtOAc extract was washed successively with water, saturated aqueous sodium bicarbonate, brine, dried over $\mathrm{MgSO}_{4}$, and evaporated under vacuum to provide a crude product which was purified by flash chromatography on silica gel with ethyl acetate/ n-hexane (1:6) to give the pyrazole-3carboxylic acid ethyl ester $\mathbf{3}$ as a white solid. Yield, m.p., elemental analyses, IR, ${ }^{1} \mathrm{H}$ NMR, ${ }^{13} \mathrm{C}$ NMR and mass spectral data are listed in tables I and II.

\section{Synthesis of 5-(4-chlorophenyl)-1-(2,4- dichlorophenyl)-4-methyl-1H-pyrazole-3- carbohydrazide (4)}

A mixture of pyrazole-3-carboxylic acid ethyl ester 3 (5 g, $12.25 \mathrm{mmol}$ ) and 80\% hydrazine hydrate $(4.7 \mathrm{~mL}, 122.5 \mathrm{mmol})$ in ethanol $(50 \mathrm{~mL})$ was heated to reflux for 5 hours. The solvent was concentrated in vacuum and the resulting residue was taken up in water, filtered, washed with water, and dried in vacuum to provide the title compound $\mathbf{4}$ as white solid. Yield, m.p., elemental analyses, IR, ${ }^{1} \mathrm{H}$ NMR, ${ }^{13} \mathrm{C}$ NMR and mass spectral data are listed in tables I and II.

General procedure A for synthesis of 2-(5(4-chlorophenyl)-1-(2,4-dichlorophenyl)-4methyl-1H-pyrazole-3-carbonyl)- $N$ subsituted hydrazine carbothioamide (5a-c)

A mixture of $4(1.5 \mathrm{~g}, 3.81 \mathrm{mmol})$ and an appropriate isothiocyanate $(3.81 \mathrm{mmol})$ in EtOH $(30 \mathrm{~mL})$ was heated at reflux for $2 \mathrm{~h}$. The solvent was concentrated in vacuum and the resulting residue was taken up in water, filtered off, washed with water, and dried to give the title compounds which were used for next step without further purification. Yields, m.p., elemental analyses, IR, ${ }^{1} \mathrm{H}$ NMR, ${ }^{13} \mathrm{C}$ NMR and mass spectral data are listed in tables I and II.

General procedure B for synthesis of 5-(5(4-chlorophenyl)-1-(2,4-dichlorophenyl)-4methyl-1 $H$-pyrazol-3-yl)-4-subsituted-4H-

\section{1,2,4-triazole-3-thiol (6a-c)}

A mixture of the appropriate thiosemicarbazides 5a-c $(4 \mathrm{mmol})$ and $2 \mathrm{~N}$ $\mathrm{NaOH}(30 \mathrm{~mL})$ was refluxed for $3 \mathrm{~h}$. After cooling, the reaction mixture was acidified to pH 6 with $2 \mathrm{M} \mathrm{HCl}$ and the resulting precipitate was filtered off and washed with water to afford a crude product which was purified by suspended in few $\mathrm{mL}$ of $\mathrm{Et}_{2} \mathrm{O}$ then the separated precipitate was filtered and washed with $\mathrm{Et}_{2} \mathrm{O}$ to give pure desired compound. Yields, m.p., elemental analyses, IR, ${ }^{1} \mathrm{H}$ NMR, ${ }^{13} \mathrm{C}$ NMR and mass spectral data are listed in tables I and II.

General procedure $C$ for synthesis of the final target compounds (7a-o)

A mixture of the appropriate 1,2,4triazole-3-thiol derivatives 6a-c $(0.5 \mathrm{mmol})$, an appropriate haloalkane $(0.6 \mathrm{mmol})$ and $\mathrm{K}_{2} \mathrm{CO}_{3}$ $(0.06 \mathrm{~g}, 0.6 \mathrm{mmol})$ in acetone $(20 \mathrm{~mL})$ was heated under reflux for $1 \mathrm{~h}$. After removal of the organic solvent in vacuo and addition of water, the resulting residue was extracted by EtOAc. The organic layer was washed with brine, dried over anhydrous $\mathrm{MgSO}_{4}$ and evaporated under reduced pressure to afford the crude product which was purified by column chromatography on silica using EtOAc/hexane (1:2) to give the desired compound. Yields, m.p., elemental analyses, IR, ${ }^{1} \mathrm{H}$ NMR, ${ }^{13} \mathrm{C}$ NMR and mass spectral data are listed in tables I and II. 
<smiles>CCOC(=O)c1nn(-c2ccc(CC(C)(C)C)cc2C)c(-c2ccc(C)cc2)c1C</smiles>

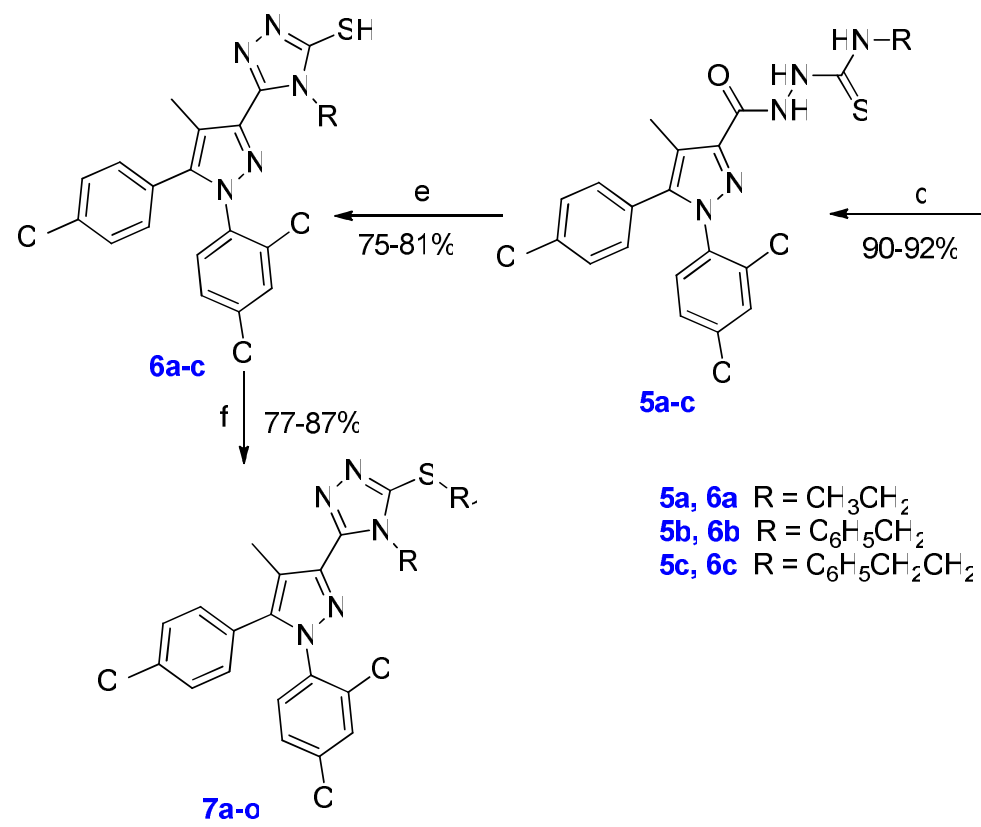

Reagents and conditions: (a) diethyl oxalate, LHMDS, $-78^{\circ} \mathrm{C}$ to room temp, $16 \mathrm{~h}$; (b) 2,4dichlorophenylhydrazine hydrochloride, EtOH, room temp, $20 \mathrm{~h}$, then acetic acid, reflux, $24 \mathrm{~h}$; (c) $\mathrm{NH}_{2} \mathrm{NH}_{2} . \mathrm{H}_{2} \mathrm{O}(85 \%)$, ethanol, reflux, $5 \mathrm{~h}$; (d) an appropriate isothiocyanate, ethanol, reflux, $2 \mathrm{~h}$; (e) $2 \mathrm{~N}$ $\mathrm{NaOH}$, reflux, $3 \mathrm{~h}$; (f) an appropriate haloalkane, $\mathrm{K}_{2} \mathrm{CO}_{3}$, acetone, reflux, $1 \mathrm{~h}$.

\begin{tabular}{|c|l|l|c|l|l||}
\hline \hline Compd. & \multicolumn{1}{|c|}{$\mathrm{R}$} & \multicolumn{1}{c|}{$\mathrm{R}_{1}$} & Compd. & \multicolumn{1}{c|}{$\mathrm{R}$} & \multicolumn{1}{c|}{$\mathrm{R}_{1}$} \\
\hline $\mathbf{7 a}$ & $\mathrm{CH}_{3} \mathrm{CH}_{2}$ & $\mathrm{C}_{6} \mathrm{H}_{5} \mathrm{CH}_{2}$ & $\mathbf{7 i}$ & $\mathrm{C}_{6} \mathrm{H}_{5} \mathrm{CH}_{2}$ & $p-\mathrm{OCH}_{3} \mathrm{C}_{6} \mathrm{H}_{4} \mathrm{CH}_{2}$ \\
\hline $\mathbf{7 b}$ & $\mathrm{CH}_{3} \mathrm{CH}_{2}$ & $\mathrm{C}_{6} \mathrm{H}_{5} \mathrm{CH}_{2} \mathrm{CH}_{2}$ & $\mathbf{7 j}$ & $\mathrm{C}_{6} \mathrm{H}_{5} \mathrm{CH}_{2}$ & $p-\mathrm{CH}_{3} \mathrm{C}_{6} \mathrm{H}_{4} \mathrm{CH}_{2}$ \\
\hline $\mathbf{7 c}$ & $\mathrm{CH}_{3} \mathrm{CH}_{2}$ & $\mathrm{C}_{6} \mathrm{H}_{5} \mathrm{CH}_{2} \mathrm{CH}_{2} \mathrm{CH}_{2}$ & $\mathbf{7 k}$ & $\mathrm{C}_{6} \mathrm{H}_{5} \mathrm{CH}_{2} \mathrm{CH}_{2}$ & $\mathrm{C}_{6} \mathrm{H}_{5} \mathrm{CH}_{2}$ \\
\hline $\mathbf{7 d}$ & $\mathrm{CH}_{3} \mathrm{CH}_{2}$ & $p-\mathrm{OCH}_{3} \mathrm{C}_{6} \mathrm{H}_{4} \mathrm{CH}_{2}$ & $\mathbf{7 l}$ & $\mathrm{C}_{6} \mathrm{H}_{5} \mathrm{CH}_{2} \mathrm{CH}_{2}$ & $\mathrm{C}_{6} \mathrm{H}_{5} \mathrm{CH}_{2} \mathrm{CH}_{2}$ \\
\hline $\mathbf{7 e}$ & $\mathrm{CH}_{3} \mathrm{CH}_{2}$ & $p-\mathrm{CH}_{3} \mathrm{C}_{6} \mathrm{H}_{4} \mathrm{CH}_{2}$ & $\mathbf{7 m}$ & $\mathrm{C}_{6} \mathrm{H}_{5} \mathrm{CH}_{2} \mathrm{CH}_{2}$ & $\mathrm{C}_{6} \mathrm{H}_{5} \mathrm{CH}_{2} \mathrm{CH}_{2} \mathrm{CH}_{2}$ \\
\hline $\mathbf{7 f}$ & $\mathrm{C}_{6} \mathrm{H}_{5} \mathrm{CH}_{2}$ & $\mathrm{C}_{6} \mathrm{H}_{5} \mathrm{CH}_{2}$ & $\mathbf{7 n}$ & $\mathrm{C}_{6} \mathrm{H}_{5} \mathrm{CH}_{2} \mathrm{CH}_{2}$ & $p-\mathrm{OCH}_{3} \mathrm{C}_{6} \mathrm{H}_{4} \mathrm{CH}_{2}$ \\
\hline $\mathbf{7 g}$ & $\mathrm{C}_{6} \mathrm{H}_{5} \mathrm{CH}_{2}$ & $\mathrm{C}_{6} \mathrm{H}_{5} \mathrm{CH}_{2} \mathrm{CH}_{2}$ & $\mathbf{7 o}$ & $\mathrm{C}_{6} \mathrm{H}_{5} \mathrm{CH}_{2} \mathrm{CH}_{2}$ & $p-\mathrm{CH}_{3} \mathrm{C}_{6} \mathrm{H}_{4} \mathrm{CH}_{2}$ \\
\hline $\mathbf{7 h}$ & $\mathrm{C}_{6} \mathrm{H}_{5} \mathrm{CH}_{2}$ & $\mathrm{C}_{6} \mathrm{H}_{5} \mathrm{CH}_{2} \mathrm{CH}_{2} \mathrm{CH}_{2}$ & - & - & - \\
\hline
\end{tabular}

Scheme 1: Synthetic route of target compounds 3-(1H-pyrazol-3-yl)-4H-1,2,4-triazoles 7a-o. 
Table I: Physicochemical properties of compounds 3, 4, 5a-c, 6a-c and 7a-o.

\begin{tabular}{|c|c|c|c|c|c|}
\hline Compd. & $\mathrm{R}$ & $\mathrm{R}^{1}$ & $\begin{array}{l}\text { Yield } \\
(\%)\end{array}$ & m.p. $\left({ }^{\circ} \mathrm{C}\right)$ & M.F. (M.Wt.) \\
\hline 3 & -- & -- & 55 & $120-121$ & $\begin{array}{c}\mathrm{C}_{19} \mathrm{H}_{15} \mathrm{C}_{13} \mathrm{~N}_{2} \mathrm{O}_{2} \\
(409.69) \\
\end{array}$ \\
\hline 4 & -- & -- & 93 & $102-104$ & $\begin{array}{c}\mathrm{C}_{17} \mathrm{H}_{13} \mathrm{Cl}_{3} \mathrm{~N}_{4} \mathrm{O} \\
(395.67)\end{array}$ \\
\hline $5 \mathbf{a}$ & $\mathrm{CH}_{3} \mathrm{CH}_{2}$ & -- & 92 & $143-145$ & $\begin{array}{c}\mathrm{C}_{20} \mathrm{H}_{18} \mathrm{Cl}_{3} \mathrm{~N}_{5} \mathrm{OS} \\
(482.81) \\
\end{array}$ \\
\hline $5 b$ & $\mathrm{C}_{6} \mathrm{H}_{5} \mathrm{CH}_{2}$ & -- & 90 & $138-140$ & $\begin{array}{c}\mathrm{C}_{25} \mathrm{H}_{20} \mathrm{Cl}_{3} \mathrm{~N}_{5} \mathrm{OS} \\
(544.88)\end{array}$ \\
\hline $5 c$ & $\mathrm{C}_{6} \mathrm{H}_{5} \mathrm{CH}_{2} \mathrm{CH}_{2}$ & -- & 91 & $125-127$ & $\begin{array}{c}\mathrm{C}_{26} \mathrm{H}_{22} \mathrm{Cl}_{3} \mathrm{~N}_{5} \mathrm{OS} \\
(558.91)\end{array}$ \\
\hline $6 \mathbf{a}$ & $\mathrm{CH}_{3} \mathrm{CH}_{2}$ & -- & 81 & $234-236$ & $\begin{array}{c}\mathrm{C}_{20} \mathrm{H}_{16} \mathrm{Cl}_{3} \mathrm{~N}_{5} \mathrm{~S} \\
(464.80)\end{array}$ \\
\hline $6 \mathbf{b}$ & $\mathrm{C}_{6} \mathrm{H}_{5} \mathrm{CH}_{2}$ & -- & 80 & $242-244$ & $\begin{array}{c}\mathrm{C}_{25} \mathrm{H}_{18} \mathrm{Cl}_{3} \mathrm{~N}_{5} \mathrm{~S} \\
(526.87) \\
\end{array}$ \\
\hline $6 c$ & $\mathrm{C}_{6} \mathrm{H}_{5} \mathrm{CH}_{2} \mathrm{CH}_{2}$ & -- & 75 & $224-226$ & $\begin{array}{c}\mathrm{C}_{26} \mathrm{H}_{20} \mathrm{Cl}_{3} \mathrm{~N}_{5} \mathrm{~S} \\
(540.89)\end{array}$ \\
\hline $7 \mathbf{a}$ & $\mathrm{CH}_{3} \mathrm{CH}_{2}$ & $\mathrm{C}_{6} \mathrm{H}_{5} \mathrm{CH}_{2}$ & 87 & $72-74$ & $\begin{array}{c}\mathrm{C}_{27} \mathrm{H}_{22} \mathrm{Cl}_{3} \mathrm{~N}_{5} \mathrm{~S} \\
(554.92) \\
\end{array}$ \\
\hline $7 b$ & $\mathrm{CH}_{3} \mathrm{CH}_{2}$ & $\mathrm{C}_{6} \mathrm{H}_{5} \mathrm{CH}_{2} \mathrm{CH}_{2}$ & 86 & $130-132$ & $\begin{array}{c}\mathrm{C}_{28} \mathrm{H}_{24} \mathrm{Cl}_{3} \mathrm{~N}_{5} \mathrm{~S} \\
(568.95)\end{array}$ \\
\hline $7 c$ & $\mathrm{CH}_{3} \mathrm{CH}_{2}$ & $\mathrm{C}_{6} \mathrm{H}_{5} \mathrm{CH}_{2} \mathrm{CH}_{2} \mathrm{CH}_{2}$ & 77 & oil & $\begin{array}{c}\mathrm{C}_{29} \mathrm{H}_{26} \mathrm{Cl}_{3} \mathrm{~N}_{5} \mathrm{~S} \\
(582.97) \\
\end{array}$ \\
\hline $7 d$ & $\mathrm{CH}_{3} \mathrm{CH}_{2}$ & $p-\mathrm{OCH}_{3} \mathrm{C}_{6} \mathrm{H}_{4} \mathrm{CH}_{2}$ & 86 & oil & $\begin{array}{c}\mathrm{C}_{28} \mathrm{H}_{24} \mathrm{Cl}_{3} \mathrm{~N}_{5} \mathrm{OS} \\
(584.95) \\
\end{array}$ \\
\hline $7 e$ & $\mathrm{CH}_{3} \mathrm{CH}_{2}$ & $p-\mathrm{CH}_{3} \mathrm{C}_{6} \mathrm{H}_{4} \mathrm{CH}_{2}$ & 88 & $90-92$ & $\begin{array}{c}\mathrm{C}_{28} \mathrm{H}_{24} \mathrm{Cl}_{3} \mathrm{~N}_{5} \mathrm{~S} \\
(568.95) \\
\end{array}$ \\
\hline $7 f$ & $\mathrm{C}_{6} \mathrm{H}_{5} \mathrm{CH}_{2}$ & $\mathrm{C}_{6} \mathrm{H}_{5} \mathrm{CH}_{2}$ & 87 & $65-67$ & $\begin{array}{c}\mathrm{C}_{32} \mathrm{H}_{24} \mathrm{Cl}_{3} \mathrm{~N}_{5} \mathrm{~S} \\
(616.99) \\
\end{array}$ \\
\hline $7 \mathrm{~g}$ & $\mathrm{C}_{6} \mathrm{H}_{5} \mathrm{CH}_{2}$ & $\mathrm{C}_{6} \mathrm{H}_{5} \mathrm{CH}_{2} \mathrm{CH}_{2}$ & 82 & $61-62$ & $\begin{array}{c}\mathrm{C}_{33} \mathrm{H}_{26} \mathrm{Cl}_{3} \mathrm{~N}_{5} \mathrm{~S} \\
(631.02) \\
\end{array}$ \\
\hline $7 \mathrm{~h}$ & $\mathrm{C}_{6} \mathrm{H}_{5} \mathrm{CH}_{2}$ & $\mathrm{C}_{6} \mathrm{H}_{5} \mathrm{CH}_{2} \mathrm{CH}_{2} \mathrm{CH}_{2}$ & 78 & oil & $\begin{array}{c}\mathrm{C}_{34} \mathrm{H}_{28} \mathrm{Cl}_{3} \mathrm{~N}_{5} \mathrm{~S} \\
(645.04)\end{array}$ \\
\hline $7 \mathbf{i}$ & $\mathrm{C}_{6} \mathrm{H}_{5} \mathrm{CH}_{2}$ & $p-\mathrm{OCH}_{3} \mathrm{C}_{6} \mathrm{H}_{4} \mathrm{CH}_{2}$ & 87 & $132-134$ & $\begin{array}{c}\mathrm{C}_{33} \mathrm{H}_{26} \mathrm{Cl}_{3} \mathrm{~N}_{5} \mathrm{OS} \\
(647.02) \\
\end{array}$ \\
\hline $7 \mathbf{j}$ & $\mathrm{C}_{6} \mathrm{H}_{5} \mathrm{CH}_{2}$ & $p-\mathrm{CH}_{3} \mathrm{C}_{6} \mathrm{H}_{4} \mathrm{CH}_{2}$ & 85 & $72-74$ & $\begin{array}{c}\mathrm{C}_{33} \mathrm{H}_{26} \mathrm{C}_{13} \mathrm{~N}_{5} \mathrm{~S} \\
(631.02) \\
\end{array}$ \\
\hline $7 k$ & $\mathrm{C}_{6} \mathrm{H}_{5} \mathrm{CH}_{2} \mathrm{CH}_{2}$ & $\mathrm{C}_{6} \mathrm{H}_{5} \mathrm{CH}_{2}$ & 86 & $59-60$ & $\begin{array}{c}\mathrm{C}_{33} \mathrm{H}_{26} \mathrm{Cl}_{3} \mathrm{~N}_{5} \mathrm{~S} \\
(631.02) \\
\end{array}$ \\
\hline 71 & $\mathrm{C}_{6} \mathrm{H}_{5} \mathrm{CH}_{2} \mathrm{CH}_{2}$ & $\mathrm{C}_{6} \mathrm{H}_{5} \mathrm{CH}_{2} \mathrm{CH}_{2}$ & 78 & $90-92$ & $\begin{array}{c}\mathrm{C}_{34} \mathrm{H}_{28} \mathrm{Cl}_{3} \mathrm{~N}_{5} \mathrm{~S} \\
(645.04) \\
\end{array}$ \\
\hline $7 \mathrm{~m}$ & $\mathrm{C}_{6} \mathrm{H}_{5} \mathrm{CH}_{2} \mathrm{CH}_{2}$ & $\mathrm{C}_{6} \mathrm{H}_{5} \mathrm{CH}_{2} \mathrm{CH}_{2} \mathrm{CH}_{2}$ & 78 & $118-120$ & $\begin{array}{c}\mathrm{C}_{35} \mathrm{H}_{30} \mathrm{Cl}_{3} \mathrm{~N}_{5} \mathrm{~S} \\
(659.07)\end{array}$ \\
\hline $7 n$ & $\mathrm{C}_{6} \mathrm{H}_{5} \mathrm{CH}_{2} \mathrm{CH}_{2}$ & $p-\mathrm{OCH}_{3} \mathrm{C}_{6} \mathrm{H}_{4} \mathrm{CH}_{2}$ & 86 & $163-165$ & $\begin{array}{c}\mathrm{C}_{34} \mathrm{H}_{28} \mathrm{Cl}_{3} \mathrm{~N}_{5} \mathrm{OS} \\
(661.04) \\
\end{array}$ \\
\hline 70 & $\mathrm{C}_{6} \mathrm{H}_{5} \mathrm{CH}_{2} \mathrm{CH}_{2}$ & $p-\mathrm{CH}_{3} \mathrm{C}_{6} \mathrm{H}_{4} \mathrm{CH}_{2}$ & 83 & $58-60$ & $\begin{array}{c}\mathrm{C}_{34} \mathrm{H}_{28} \mathrm{Cl}_{3} \mathrm{~N}_{5} \mathrm{~S} \\
(645.04)\end{array}$ \\
\hline
\end{tabular}

Table II: Spectral characterization of compounds 3, 4, 5a-c, 6a-c and 7a-o.

\begin{tabular}{|c|c|c|c|}
\hline Compd. & ${ }^{1} \mathrm{H}$ NMR $(\delta \mathrm{ppm})$ & ${ }^{13} \mathrm{C}$ NMR $(\delta \mathrm{ppm})$ & $\begin{array}{l}\text { HRMES } \\
\text { (calculated } \\
\text { for }[\mathrm{M}+\mathrm{H}]^{+} \\
\text {/found) }\end{array}$ \\
\hline 3 & $\begin{array}{l}\left(400 \mathrm{MHz}, \mathrm{CDCl}_{3}\right) \delta 7.35(\mathrm{~d}, J=2.3 \mathrm{~Hz}, 1 \mathrm{H}, \\
\mathrm{Ar}-\mathrm{H}), 7.33(\mathrm{~d}, J=8.5 \mathrm{~Hz}, 1 \mathrm{H}, \mathrm{Ar}-\mathrm{H}), 7.29- \\
7.24(\mathrm{~m}, 3 \mathrm{H}, \mathrm{Ar}-\mathrm{H}), 7.05(\mathrm{~d}, J=8.8 \mathrm{~Hz}, 2 \mathrm{H}, \mathrm{Ar}- \\
\mathrm{H}), 4.42\left(\mathrm{q}, J=7.1 \mathrm{~Hz}, 2 \mathrm{H}, \mathrm{OCH}_{2} \mathrm{CH}_{3}\right), 2.31(\mathrm{~s},\end{array}$ & $\begin{array}{l}\left(101 \mathrm{MHz}, \mathrm{CDCl}_{3}\right) \delta 162.71,142.94, \\
142.85,136.01,135.88,134.97,133.01, \\
130.86,130.72,130.06,128.86,127.75, \\
127.02,119.11,60.95,14.42,9.66 .\end{array}$ & $\begin{array}{l}409.0272 \\
409.0277\end{array}$ \\
\hline
\end{tabular}




\begin{tabular}{|c|c|c|c|}
\hline $\begin{array}{l}\text { Table II: } \\
\text { Compd. }\end{array}$ & ${ }^{1} \mathrm{H}$ NMR $(\delta$ ppm) & ${ }^{13} \mathrm{C}$ NMR $(\delta \mathrm{ppm})$ & $\begin{array}{l}\text { HRMES } \\
\text { (calculated } \\
\text { for }[\mathrm{M}+\mathrm{H}]^{+} \\
\text {/found) }\end{array}$ \\
\hline & $\left.3 \mathrm{H}, \mathrm{CH}_{3}\right), 1.39\left(\mathrm{t}, J=7.1 \mathrm{~Hz}, 3 \mathrm{H}, \mathrm{OCH}_{2} \mathrm{CH}_{3}\right)$. & & \\
\hline 4 & $\begin{array}{l}\left(400 \mathrm{MHz}, \mathrm{DMSO}-d_{6}\right) \delta 9.46(\mathrm{~s}, 1 \mathrm{H}, \mathrm{NH}) \\
7.77-7.67(\mathrm{~m}, 2 \mathrm{H}, \mathrm{Ar}-\mathrm{H}), 7.56(\mathrm{dd}, J=8.7,2.3 \\
\mathrm{Hz}, 1 \mathrm{H}, \mathrm{Ar}-\mathrm{H}), 7.44(\mathrm{~d}, J=8.2 \mathrm{~Hz}, 2 \mathrm{H}, \mathrm{Ar}-\mathrm{H}), \\
7.23(\mathrm{~d}, J=8.1 \mathrm{~Hz}, 2 \mathrm{H}, \mathrm{Ar}-\mathrm{H}), 4.15(\mathrm{~s}, 2 \mathrm{H}, \\
\left.\mathrm{NH}_{2}\right), 2.24\left(\mathrm{~s}, 3 \mathrm{H}, \mathrm{CH}_{3}\right) .\end{array}$ & $\begin{array}{l}\left(101 \mathrm{MHz}, \mathrm{DMSO}-d_{6}\right) \delta 161.51,144.27, \\
142.20,135.78,135.00,133.72,132.09, \\
131.84,131.21,129.62,128.74,128.32, \\
127.23,116.19,9.00 .\end{array}$ & $\begin{array}{l}395.0228 \\
395.0216\end{array}$ \\
\hline $5 \mathbf{5 a}$ & $\begin{array}{l}\left(400 \mathrm{MHz}, \mathrm{DMSO}-d_{6}\right) \delta 10.07(\mathrm{~s}, 1 \mathrm{H}, \\
\mathrm{NHNHCSNH}), 9.22(\mathrm{~s}, 1 \mathrm{H}, \mathrm{NHN} \underline{\mathrm{HCSNH}}), 7.96 \\
(\mathrm{t}, J=5.7 \mathrm{~Hz}, 1 \mathrm{H}, \mathrm{NHNHCSN} \underline{\mathrm{H}}), 7.77(\mathrm{~d}, J= \\
2.3 \mathrm{~Hz}, 1 \mathrm{H}, \mathrm{Ar}-\mathrm{H}), 7.70(\mathrm{~d}, J=8.5 \mathrm{~Hz}, 1 \mathrm{H}, \mathrm{Ar}- \\
\mathrm{H}), 7.60(\mathrm{dd}, J=8.5,2.3 \mathrm{~Hz}, 1 \mathrm{H}, \mathrm{Ar}-\mathrm{H}), 7.46 \\
(\mathrm{~d}, J=8.2 \mathrm{~Hz}, 2 \mathrm{H}, \mathrm{Ar}-\mathrm{H}), 7.23(\mathrm{~d}, J=8.3 \mathrm{~Hz}, \\
2 \mathrm{H}, \mathrm{Ar}-\mathrm{H}), 3.47\left(\mathrm{p}, J=7.2 \mathrm{~Hz}, 2 \mathrm{H}, \mathrm{C}_{2} \mathrm{CH}_{3}\right), \\
2.25\left(\mathrm{~s}, 3 \mathrm{H}, \mathrm{CH}_{3}\right), 1.06(\mathrm{t}, J=7.2 \mathrm{~Hz}, 3 \mathrm{H}, \\
\left.\mathrm{CH}_{2} \mathrm{CH}_{3}\right) .\end{array}$ & $\begin{array}{l}\left(101 \mathrm{MHz}, \mathrm{DMSO}-d_{6}\right) \delta 189.08,161.62, \\
143.56,142.41,135.64,135.13,133.84, \\
131.89,131.83,131.19,129.68,128.81, \\
128.42,127.06,117.27,38.56,14.49,9.13 .\end{array}$ & $\begin{array}{l}482.0370 \\
482.0358\end{array}$ \\
\hline 5b & $\begin{array}{l}\left(400 \mathrm{MHz}, \mathrm{DMSO}-d_{6}\right) \delta 10.26(\mathrm{~s}, 1 \mathrm{H}, \\
\mathrm{NHNHCSNH}), 9.47(\mathrm{~s}, 1 \mathrm{H}, \mathrm{NHNHCSNH}), 8.54 \\
(\mathrm{t}, J=7.9 \mathrm{~Hz}, 1 \mathrm{H}, \mathrm{NHNHCSN} \underline{\mathrm{H}}), 7.77-7.67(\mathrm{~m}, \\
2 \mathrm{H}, \mathrm{Ar}-\mathrm{H}), 7.59(\mathrm{dd}, J=8.5,2.3 \mathrm{~Hz}, 1 \mathrm{H}, \mathrm{Ar}-\mathrm{H}), \\
7.45(\mathrm{~d}, J=8.2 \mathrm{~Hz}, 2 \mathrm{H}, \mathrm{Ar}-\mathrm{H}), 7.38-7.16(\mathrm{~m}, \\
7 \mathrm{H}, \mathrm{Ar}-\mathrm{H}), 4.77\left(\mathrm{~d}, J=5.4 \mathrm{~Hz}, 2 \mathrm{H}, \mathrm{NHC}_{2}\right), \\
2.28\left(\mathrm{~s}, 3 \mathrm{H}, \mathrm{CH}_{3}\right) .\end{array}$ & $\begin{array}{l}\left(101 \mathrm{MHz}, \mathrm{DMSO}-d_{6}\right) \delta 190.30,143.64, \\
142.45,139.44,138.12,135.65,135.19, \\
133.91,131.92,131.21,129.69,128.84, \\
128.27,127.98,127.37,127.12,127.00, \\
126.54,117.36,47.76,9.13 .\end{array}$ & $\begin{array}{l}544.0527 \\
544.0511\end{array}$ \\
\hline $5 c$ & $\begin{array}{l}\left(400 \mathrm{MHz}, \mathrm{DMSO}-d_{6}\right) \delta 10.13(\mathrm{~s}, 1 \mathrm{H}, \\
\mathrm{NHNHCSNH}), 9.34(\mathrm{~s}, 1 \mathrm{H}, \mathrm{NHNHCSNH}), 8.03 \\
(\mathrm{t}, J=5.8 \mathrm{~Hz}, 1 \mathrm{H}, \mathrm{NHNHCSNH}), 7.78(\mathrm{~d}, J= \\
2.1 \mathrm{~Hz}, 1 \mathrm{H}, \mathrm{Ar}-\mathrm{H}), 7.71(\mathrm{dd}, J=8.6,1.6 \mathrm{~Hz}, \\
1 \mathrm{H}, \mathrm{Ar}-\mathrm{H}), 7.61(\mathrm{dd}, J=8.5,2.0 \mathrm{~Hz}, 1 \mathrm{H}, \mathrm{Ar}-\mathrm{H}), \\
7.47(\mathrm{dd}, J=8.5,1.8 \mathrm{~Hz}, 2 \mathrm{H}, \mathrm{Ar}-\mathrm{H}), 7.31-7.16 \\
(\mathrm{~m}, 7 \mathrm{H}, \mathrm{Ar}-\mathrm{H}), 3.64(\mathrm{q}, J=7.5 \mathrm{~Hz}, 2 \mathrm{H}, \\
\left.\mathrm{NHC}_{2} \underline{\mathrm{CH}}_{2}\right), 2.82(\mathrm{t}, J=7.5 \mathrm{~Hz}, 2 \mathrm{H}, \\
\left.\mathrm{NHCH}_{2} \underline{\mathrm{CH}}_{2}\right), 2.27\left(\mathrm{~s}, 3 \mathrm{H}, \mathrm{CH}_{3}\right) .\end{array}$ & $\begin{array}{l}\left(101 \mathrm{MHz}, \mathrm{DMSO}-d_{6}\right) \delta 143.50,142.42, \\
139.37,135.62,135.14,133.84,131.89, \\
131.82,131.20,129.69,128.82,128.62, \\
128.43,128.39,128.35,127.04,126.05 \\
117.28,109.53,45.34,34.91,9.14\end{array}$ & $\begin{array}{l}558.0683 \\
558.0667\end{array}$ \\
\hline 6a & $\begin{array}{l}\left(400 \mathrm{MHz}, \mathrm{DMSO}-d_{6}\right) \delta 7.81(\mathrm{~d}, J=2.2 \mathrm{~Hz}, \\
1 \mathrm{H}, \mathrm{Ar}-\mathrm{H}), 7.66(\mathrm{~d}, J=8.6 \mathrm{~Hz}, 1 \mathrm{H}, \mathrm{Ar}-\mathrm{H}), 7.55 \\
(\mathrm{dd}, J=8.5,2.3 \mathrm{~Hz}, 1 \mathrm{H}, \mathrm{Ar}-\mathrm{H}), 7.47(\mathrm{~d}, J=8.2 \\
\mathrm{Hz}, 2 \mathrm{H}, \mathrm{Ar}-\mathrm{H}), 7.26(\mathrm{~d}, J=8.2 \mathrm{~Hz}, 2 \mathrm{H}, \mathrm{Ar}-\mathrm{H}), \\
4.36\left(\mathrm{q}, J=7.0 \mathrm{~Hz}, 2 \mathrm{H}, \mathrm{C}_{2}{ }_{2} \mathrm{CH}_{3}\right), 2.23(\mathrm{~s}, 3 \mathrm{H}, \\
\left.\mathrm{CH}_{3}\right), 1.21\left(\mathrm{t}, J=7.0 \mathrm{~Hz}, 3 \mathrm{H}, \mathrm{CH}_{2} \mathrm{CH}_{3}\right) .\end{array}$ & $\begin{array}{l}\left(101 \mathrm{MHz}, \mathrm{DMSO}-d_{6}\right) \delta 166.90,144.57 \\
141.93,139.74,135.75,134.94,133.84 \\
132.04,131.54,131.27,129.88,128.82 \\
128.42,127.07,115.72,39.68,13.80,9.65\end{array}$ & $\begin{array}{l}464.0265 \\
464.0251\end{array}$ \\
\hline $\mathbf{6 b}$ & $\begin{array}{l}\left(400 \mathrm{MHz}, \mathrm{DMSO}-d_{6}\right) \delta 7.78(\mathrm{~s}, 1 \mathrm{H}, \mathrm{Ar}-\mathrm{H}), \\
7.58(\mathrm{~s}, 2 \mathrm{H}, \mathrm{Ar}-\mathrm{H}), 7.45(\mathrm{~d}, J=8.2 \mathrm{~Hz}, 2 \mathrm{H}, \mathrm{Ar}- \\
\mathrm{H}), 7.26-7.17(\mathrm{~m}, 7 \mathrm{H}, \mathrm{Ar}-\mathrm{H}), 5.63(\mathrm{~s}, 2 \mathrm{H}, \\
\left.\mathrm{NCH}_{2}\right), 2.14\left(\mathrm{~s}, 3 \mathrm{H}, \mathrm{CH}_{3}\right) .\end{array}$ & $\begin{array}{l}\left(101 \mathrm{MHz}, \mathrm{DMSO}-d_{6}\right) \delta 167.82,144.91, \\
142.11,138.78,136.21,135.48,135.13, \\
133.96,131.86,131.53,131.23,129.80, \\
128.82,128.44,128.16,127.41,127.31 \\
126.75,115.99,47.20,9.45 .\end{array}$ & $\begin{array}{l}526.0421 \\
526.0405\end{array}$ \\
\hline 6c & $\begin{array}{l}\left(400 \mathrm{MHz}, \mathrm{DMSO}-d_{6}\right) \delta 7.84(\mathrm{~d}, J=2.2 \mathrm{~Hz}, \\
1 \mathrm{H}, \mathrm{Ar}-\mathrm{H}), 7.73(\mathrm{~d}, J=8.5 \mathrm{~Hz}, 1 \mathrm{H}, \mathrm{Ar}-\mathrm{H}), 7.62 \\
(\mathrm{dd}, J=8.5,2.3 \mathrm{~Hz}, 1 \mathrm{H}, \mathrm{Ar}-\mathrm{H}), 7.48(\mathrm{~d}, J=8.1 \\
\mathrm{Hz}, 2 \mathrm{H}, \mathrm{Ar}-\mathrm{H}), 7.26(\mathrm{~d}, J=8.1 \mathrm{~Hz}, 2 \mathrm{H}, \mathrm{Ar}-\mathrm{H}), \\
7.16-7.12(\mathrm{~m}, 3 \mathrm{H}, \mathrm{Ar}-\mathrm{H}), 7.06-6.99(\mathrm{~m}, 2 \mathrm{H}, \\
\mathrm{Ar}-\mathrm{H}), 4.57\left(\mathrm{t}, J=7.5 \mathrm{~Hz}, 2 \mathrm{H}, \mathrm{NC}_{2} \mathrm{CH}_{2}\right), 3.01 \\
\left(\mathrm{t}, J=7.5 \mathrm{~Hz}, 2 \mathrm{H}, \mathrm{NCH}_{2} \mathrm{CH}_{2}\right), 2.09(\mathrm{~s}, 3 \mathrm{H}, \\
\left.\mathrm{CH}_{3}\right) .\end{array}$ & $\begin{array}{l}\left(101 \mathrm{MHz}, \mathrm{DMSO}-d_{6}\right) \delta 166.83,144.86, \\
142.11,138.83,137.67,135.64,135.21, \\
133.98,132.03,131.67,131.21,129.84, \\
128.89,128.63,128.50,128.24,126.86, \\
126.43,115.93,45.82,33.45,9.44 .\end{array}$ & $\begin{array}{l}540.0578 \\
540.0560\end{array}$ \\
\hline $7 \mathbf{a}$ & $\begin{array}{l}\left(400 \mathrm{MHz}, \mathrm{CDCl}_{3}\right) \delta 7.33(\mathrm{~d}, J=2.3 \mathrm{~Hz}, 1 \mathrm{H}, \\
\mathrm{Ar}-\mathrm{H}), 7.29(\mathrm{~d}, J=7.0 \mathrm{~Hz}, 2 \mathrm{H}, \mathrm{Ar}-\mathrm{H}), 7.21-\end{array}$ & $\begin{array}{l}\left(101 \mathrm{MHz}, \mathrm{CDCl}_{3}\right) \delta 150.74,149.11, \\
141.94,140.82,136.76,136.08,135.51\end{array}$ & $\begin{array}{l}554.0734 \\
554.0721\end{array}$ \\
\hline
\end{tabular}


Table II: Continued.

\begin{tabular}{|c|c|c|c|}
\hline Compd. & ${ }^{1} \mathrm{H}$ NMR $(\delta$ ppm) & ${ }^{13} \mathrm{C}$ NMR $(\delta \mathrm{ppm})$ & $\begin{array}{l}\text { HRMES } \\
\text { (calculated } \\
\text { for }[\mathrm{M}+\mathrm{H}]^{+} \\
\text {/found) }\end{array}$ \\
\hline & $\begin{array}{l}7.12(\mathrm{~m}, 6 \mathrm{H}, \mathrm{Ar}-\mathrm{H}), 7.07(\mathrm{~d}, J=8.5 \mathrm{~Hz}, 1 \mathrm{H}, \mathrm{Ar}- \\
\mathrm{H}), 7.00(\mathrm{~d}, J=8.3 \mathrm{~Hz}, 2 \mathrm{H}, \mathrm{Ar}-\mathrm{H}), 4.43(\mathrm{~s}, 2 \mathrm{H}, \\
\left.\mathrm{SCH}_{2}\right), 4.17\left(\mathrm{q}, J=7.1 \mathrm{~Hz}, 2 \mathrm{H}, \mathrm{CH}_{2} \mathrm{CH}_{3}\right), 2.33 \\
\left(\mathrm{~s}, 3 \mathrm{H}, \mathrm{CH}_{3}\right), 1.12\left(\mathrm{t}, J=7.1 \mathrm{~Hz}, 3 \mathrm{H}, \mathrm{CH}_{2} \mathrm{CH}_{3}\right) .\end{array}$ & $\begin{array}{l}\text { 134.79, 132.87, 130.79, 130.38, 130.32, } \\
\text { 129.06, 128.88, 128.61, 127.78, 127.67, } \\
127.34,117.03,40.49,37.77,15.09,10.12 .\end{array}$ & \\
\hline $7 b$ & $\begin{array}{l}\left(400 \mathrm{MHz}, \mathrm{CDCl}_{3}\right) \delta 7.35(\mathrm{~d}, J=2.3 \mathrm{~Hz}, 1 \mathrm{H}, \\
\mathrm{Ar}-\mathrm{H}), 7.24-7.05(\mathrm{~m}, 9 \mathrm{H}, \mathrm{Ar}-\mathrm{H}), 7.05-6.98(\mathrm{~m}, \\
2 \mathrm{H}, \mathrm{Ar}-\mathrm{H}), 4.27\left(\mathrm{q}, J=7.1 \mathrm{~Hz}, 2 \mathrm{H}, \mathrm{C}_{2} \mathrm{CH}_{3}\right), \\
3.47\left(\mathrm{t}, J=7.7 \mathrm{~Hz}, 2 \mathrm{H}, \mathrm{SC}_{\underline{H}_{2}} \mathrm{CH}_{2}\right), 3.05(\mathrm{t}, J= \\
\left.7.7 \mathrm{~Hz}, 2 \mathrm{H}, \mathrm{SCH}_{2} \mathrm{C}_{2}\right), 2.35\left(\mathrm{~s}, 3 \mathrm{H}, \mathrm{CH}_{3}\right), 1.23 \\
\left(\mathrm{t}, J=7.1 \mathrm{~Hz}, 3 \mathrm{H}, \mathrm{CH}_{2} \mathrm{C}_{3}\right) .\end{array}$ & $\begin{array}{l}\left(101 \mathrm{MHz}, \mathrm{CDCl}_{3}\right) \delta 151.10,149.13, \\
141.98,140.90,139.67,136.15,135.55, \\
\text { 134.83, 132.94, 130.84, 130.42, 130.38, } \\
\text { 128.92, 128.76, 128.47, 127.82, 127.39, } \\
\text { 126.54, 117.05, 40.56, 35.93, 34.08, 15.16, } \\
10.19 .\end{array}$ & $\begin{array}{l}568.0891 \\
568.0876\end{array}$ \\
\hline $7 c$ & $\begin{array}{l}\left(400 \mathrm{MHz}, \mathrm{CDCl}_{3}\right) \delta 7.34(\mathrm{~d}, J=2.7 \mathrm{~Hz}, 1 \mathrm{H}, \\
\mathrm{Ar}-\mathrm{H}), 7.23-7.03(\mathrm{~m}, 9 \mathrm{H}, \mathrm{Ar}-\mathrm{H}), 7.00(\mathrm{~d}, J= \\
8.3 \mathrm{~Hz}, 2 \mathrm{H}, \mathrm{Ar}-\mathrm{H}), 4.30(\mathrm{q}, J=7.1 \mathrm{~Hz}, 2 \mathrm{H}, \\
\left.\mathrm{C}_{2} \mathrm{CH}_{3}\right), 3.23(\mathrm{t}, J=7.6 \mathrm{~Hz}, 2 \mathrm{H}, \\
\left.\mathrm{SC}_{\underline{H}_{2}} \mathrm{CH}_{2} \mathrm{CH}_{2}\right), 2.69(\mathrm{t}, J=7.6 \mathrm{~Hz}, 2 \mathrm{H}, \\
\left.\mathrm{SCH}_{2} \mathrm{CH}_{2} \mathrm{CH}_{2}\right), 2.33\left(\mathrm{~s}, 3 \mathrm{H}, \mathrm{CH}_{3}\right), 2.06(\mathrm{p}, J= \\
\left.7.6 \mathrm{~Hz}, 2 \mathrm{H}, \mathrm{SCH}_{2} \mathrm{CH}_{2} \mathrm{CH}_{2}\right), 1.24(\mathrm{t}, J=7.1 \mathrm{~Hz}, \\
\left.3 \mathrm{H}, \mathrm{CH}_{2} \mathrm{CH}_{3}\right) .\end{array}$ & $\begin{array}{l}\left(101 \mathrm{MHz}, \mathrm{CDCl}_{3}\right) \delta 151.15,149.08, \\
141.95,140.99,140.89,136.12,135.51, \\
134.79,132.91,130.81,130.40,130.34, \\
128.88,128.47,128.39,127.79,127.37, \\
125.98,117.03,40.54,34.64,32.29,31.13, \\
15.16,10.14 .\end{array}$ & $\begin{array}{l}582.1047 \\
582.1036\end{array}$ \\
\hline 7d & $\begin{array}{l}\left(400 \mathrm{MHz}, \mathrm{CDCl}_{3}\right) \delta 7.36(\mathrm{~d}, J=2.3 \mathrm{~Hz}, 1 \mathrm{H}, \\
\mathrm{Ar}-\mathrm{H}), 7.24-7.19(\mathrm{~m}, 4 \mathrm{H}, \mathrm{Ar}-\mathrm{H}), 7.15(\mathrm{dd}, J= \\
8.4,2.2 \mathrm{~Hz}, 1 \mathrm{H}, \mathrm{Ar}-\mathrm{H}), 7.08(\mathrm{~d}, J=8.4 \mathrm{~Hz}, 1 \mathrm{H}, \\
\mathrm{Ar}-\mathrm{H}), 7.02(\mathrm{~d}, J=8.1 \mathrm{~Hz}, 2 \mathrm{H}, \mathrm{Ar}-\mathrm{H}), 6.76- \\
6.69(\mathrm{~m}, 2 \mathrm{H}, \mathrm{Ar}-\mathrm{H}), 4.40\left(\mathrm{~s}, 2 \mathrm{H}, \mathrm{SCH}_{2}\right), 4.19(\mathrm{q}, \\
\left.J=7.1 \mathrm{~Hz}, 2 \mathrm{H}, \mathrm{C}_{2} \mathrm{CH}_{3}\right), 3.66\left(\mathrm{~s}, 3 \mathrm{H}, \mathrm{OCH}_{3}\right), \\
2.33\left(\mathrm{~s}, 3 \mathrm{H}, \mathrm{CH}_{3}\right), 1.15(\mathrm{t}, J=7.1 \mathrm{~Hz}, 3 \mathrm{H}, \\
\left.\mathrm{CH}_{2} \mathrm{CH}_{3}\right) .\end{array}$ & $\begin{array}{l}\left(101 \mathrm{MHz}, \mathrm{CDCl}_{3}\right) \delta 159.15,150.94, \\
149.08,141.99,140.86,136.13,135.57, \\
134.85,132.93,130.83,130.41,130.38, \\
130.34,128.93,128.71,127.82,127.38, \\
117.08,114.06,55.26,40.53,37.42,15.15, \\
10.14 .\end{array}$ & $\begin{array}{l}584.0840 \\
584.0828\end{array}$ \\
\hline $7 e$ & $\begin{array}{l}\left(400 \mathrm{MHz}, \mathrm{CDCl}_{3}\right) \delta 7.41(\mathrm{~d}, J=2.2 \mathrm{~Hz}, 1 \mathrm{H}, \\
\mathrm{Ar}-\mathrm{H}), 7.30-7.19(\mathrm{~m}, 5 \mathrm{H}, \mathrm{Ar}-\mathrm{H}), 7.15(\mathrm{~d}, J= \\
8.5 \mathrm{~Hz}, 1 \mathrm{H}, \mathrm{Ar}-\mathrm{H}), 7.10-7.05(\mathrm{~m}, 4 \mathrm{H}, \mathrm{Ar}-\mathrm{H}), \\
4.47\left(\mathrm{~s}, 2 \mathrm{H}, \mathrm{SCH}_{2}\right), 4.26(\mathrm{q}, J=7.1 \mathrm{~Hz}, 2 \mathrm{H}, \\
\left.\underline{\mathrm{C}}_{2} \mathrm{CH}_{3}\right), 2.41\left(\mathrm{~s}, 3 \mathrm{H}, \mathrm{CH}_{3}\right), 2.26\left(\mathrm{~s}, 3 \mathrm{H}, \mathrm{CH}_{3}\right), \\
1.21\left(\mathrm{t}, J=7.1 \mathrm{~Hz}, 3 \mathrm{H}, \mathrm{CH}_{2} \mathrm{CH}_{3}\right) .\end{array}$ & $\begin{array}{l}\left(101 \mathrm{MHz}, \mathrm{CDCl}_{3}\right) \delta 150.89,149.08, \\
141.91,140.91,137.36,136.10,135.49, \\
134.78,133.64,132.87,130.79,130.39, \\
130.31,129.29,128.98,128.88,127.78, \\
127.37,116.99,40.44,37.46,21.10,15.10, \\
10.12 .\end{array}$ & $\begin{array}{l}568.0891 \\
568.0878\end{array}$ \\
\hline $7 f$ & $\begin{array}{l}\left(400 \mathrm{MHz}, \mathrm{CDCl}_{3}\right) \delta 7.32-7.23(\mathrm{~m}, 3 \mathrm{H}, \mathrm{Ar}-\mathrm{H}), \\
7.23-7.06(\mathrm{~m}, 9 \mathrm{H}, \mathrm{Ar}-\mathrm{H}), 7.02(\mathrm{dd}, J=8.5,1.2 \\
\mathrm{Hz}, 1 \mathrm{H}, \mathrm{Ar}-\mathrm{H}), 6.99-6.96(\mathrm{~m}, 4 \mathrm{H}, \mathrm{Ar}-\mathrm{H}), 5.45 \\
\left(\mathrm{~s}, 2 \mathrm{H}, \mathrm{NCH}_{2}\right), 4.38\left(\mathrm{~s}, 2 \mathrm{H}, \mathrm{SCH}_{2}\right), 2.30(\mathrm{~s}, 3 \mathrm{H}, \\
\left.\mathrm{CH}_{3}\right) .\end{array}$ & $\begin{array}{l}\left(101 \mathrm{MHz}, \mathrm{CDCl}_{3}\right) \delta 151.82,149.45, \\
142.11,140.85,136.59,136.06,135.71, \\
135.67,134.91,132.89,130.83,130.48, \\
130.34,129.21,128.94,128.69,128.52, \\
127.83,127.78,127.45,127.33,117.30, \\
48.29,38.05,10.14 .\end{array}$ & $\begin{array}{l}616.0891 \\
616.0876\end{array}$ \\
\hline $7 \mathrm{~g}$ & $\begin{array}{l}\left(400 \mathrm{MHz}, \mathrm{CDCl}_{3}\right) \delta 7.29(\mathrm{~d}, J=2.2 \mathrm{~Hz}, 1 \mathrm{H}, \\
\mathrm{Ar}-\mathrm{H}), 7.21-7.00(\mathrm{~m}, 14 \mathrm{H}, \mathrm{Ar}-\mathrm{H}), 7.00-6.94 \\
(\mathrm{~m}, 2 \mathrm{H}, \mathrm{Ar}-\mathrm{H}), 5.54\left(\mathrm{~s}, 2 \mathrm{H}, \mathrm{NCH}_{2}\right), 3.38(\mathrm{t}, J= \\
\left.7.6 \mathrm{~Hz}, 2 \mathrm{H}, \mathrm{SC}_{2} \mathrm{CH}_{2}\right), 2.98(\mathrm{t}, J=7.6 \mathrm{~Hz}, 2 \mathrm{H}, \\
\left.\mathrm{SCH}_{2} \underline{\mathrm{C}}_{2}\right), 2.32\left(\mathrm{~s}, 3 \mathrm{H}, \mathrm{CH}_{3}\right) .\end{array}$ & $\begin{array}{l}\left(101 \mathrm{MHz}, \mathrm{CDCl}_{3}\right) \delta 152.04,149.41, \\
142.08,140.89,139.63,136.04,135.76, \\
135.63,134.87,132.87,130.82,130.46, \\
130.30,128.91,128.75,128.50,128.46, \\
127.80,127.77,127.44,127.31,126.53, \\
117.23,48.30,35.85,34.42,10.16 . \\
\end{array}$ & $\begin{array}{l}630.1047 \\
630.1034\end{array}$ \\
\hline $7 \mathrm{~h}$ & $\begin{array}{l}\left(400 \mathrm{MHz}, \mathrm{CDCl}_{3}\right) \delta 7.29(\mathrm{~d}, J=2.4 \mathrm{~Hz}, 1 \mathrm{H}, \\
\mathrm{Ar}-\mathrm{H}), 7.23-7.00(\mathrm{~m}, 14 \mathrm{H}, \mathrm{Ar}-\mathrm{H}), 7.00-6.94 \\
(\mathrm{~m}, 2 \mathrm{H}, \mathrm{Ar}-\mathrm{H}), 5.57\left(\mathrm{~s}, 2 \mathrm{H}, \mathrm{NCH}_{2}\right), 3.14(\mathrm{t}, J= \\
\left.7.6 \mathrm{~Hz}, 2 \mathrm{H}, \mathrm{SC}_{2} \underline{\mathrm{H}}_{2} \mathrm{CH}_{2} \mathrm{CH}_{2}\right), 2.63(\mathrm{t}, J=7.6 \mathrm{~Hz}, \\
\left.2 \mathrm{H}, \mathrm{SCH}_{2} \mathrm{CH}_{2} \underline{\mathrm{CH}}_{2}\right), 2.31\left(\mathrm{~s}, 3 \mathrm{H}, \mathrm{CH}_{3}\right), 2.00(\mathrm{p}, \\
\left.J=7.6 \mathrm{~Hz}, 2 \mathrm{H}, \mathrm{SCH}_{2} \mathrm{CH}_{2} \mathrm{CH}_{2}\right) .\end{array}$ & $\begin{array}{l}\left(101 \mathrm{MHz}, \mathrm{CDCl}_{3}\right) \delta 152.16,149.39, \\
142.08,141.00,140.90,136.05,135.79, \\
135.63,134.87,132.88,130.82,130.46, \\
130.31,128.91,128.51,128.41,127.80, \\
127.78,127.42,127.32,126.01,117.25, \\
48.32,34.61,32.65,31.01,10.13 .\end{array}$ & $\begin{array}{l}644.1204 \\
644.1190\end{array}$ \\
\hline
\end{tabular}


Table II: Continued.

\begin{tabular}{|c|c|c|c|}
\hline Compd. & ${ }^{1} \mathrm{H}$ NMR $(\delta \mathrm{ppm})$ & ${ }^{13} \mathrm{C}$ NMR $(\delta \mathrm{ppm})$ & $\begin{array}{c}\text { HRMES } \\
\text { (calculated } \\
\text { for }[\mathrm{M}+\mathrm{H}]^{+} \\
\text {/found) }\end{array}$ \\
\hline $7 \mathbf{i}$ & $\begin{array}{l}\left(400 \mathrm{MHz}, \mathrm{CDCl}_{3}\right) \delta 7.31(\mathrm{~d}, J=1.9 \mathrm{~Hz}, 1 \mathrm{H}, \\
\mathrm{Ar}-\mathrm{H}), 7.23-7.15(\mathrm{~m}, 5 \mathrm{H}, \mathrm{Ar}-\mathrm{H}), 7.14-7.10(\mathrm{~m}, \\
3 \mathrm{H}, \mathrm{Ar}-\mathrm{H}), 7.03(\mathrm{~d}, J=8.5 \mathrm{~Hz}, 1 \mathrm{H}, \mathrm{Ar}-\mathrm{H}), \\
6.70-6.95(\mathrm{~m}, 3 \mathrm{H}, \mathrm{Ar}-\mathrm{H}), 6.79(\mathrm{dd}, J=8.6,2.0 \\
\mathrm{Hz}, 1 \mathrm{H}, \mathrm{Ar}-\mathrm{H}), 6.72(\mathrm{dd}, J=8.3,1.8 \mathrm{~Hz}, 2 \mathrm{H}, \\
\mathrm{Ar}-\mathrm{H}), 5.46\left(\mathrm{~s}, 2 \mathrm{H}, \mathrm{NCH}_{2}\right), 4.34\left(\mathrm{~s}, 2 \mathrm{H}, \mathrm{SCH}_{2}\right), \\
3.67\left(\mathrm{~s}, 3 \mathrm{H}, \mathrm{OCH}_{3}\right), 2.30\left(\mathrm{~s}, 3 \mathrm{H}, \mathrm{CH}_{3}\right) .\end{array}$ & $\begin{array}{l}\left(101 \mathrm{MHz}, \mathrm{CDCl}_{3}\right) \delta 159.16,151.90, \\
149.30,142.08,140.75,136.00,135.65, \\
134.88,132.84,130.79,130.42,130.39, \\
130.29,128.90,128.59,128.47,127.78, \\
127.74,127.41,127.27,117.27,114.04, \\
113.87,55.26,48.26,37.72,10.05\end{array}$ & $\begin{array}{l}646.0996 \\
646.0982\end{array}$ \\
\hline $7 \mathbf{j}$ & $\begin{array}{l}\left(400 \mathrm{MHz}, \mathrm{CDCl}_{3}\right) \delta 7.30(\mathrm{~d}, J=2.2 \mathrm{~Hz}, 1 \mathrm{H} \\
\mathrm{Ar}-\mathrm{H}), 7.22-7.08(\mathrm{~m}, 8 \mathrm{H}, \mathrm{Ar}-\mathrm{H}), 7.06-6.94(\mathrm{~m}, \\
7 \mathrm{H}, \mathrm{Ar}-\mathrm{H}), 5.46\left(\mathrm{~s}, 2 \mathrm{H}, \mathrm{NCH}_{2}\right), 4.36(\mathrm{~s}, 2 \mathrm{H}, \\
\left.\mathrm{SCH}_{2}\right), 2.30\left(\mathrm{~s}, 3 \mathrm{H}, \mathrm{CH}_{3}\right), 2.21\left(\mathrm{~s}, 3 \mathrm{H}, \mathrm{CH}_{3}\right)\end{array}$ & $\begin{array}{l}\left(101 \mathrm{MHz}, \mathrm{CDCl}_{3}\right) \delta 151.97,149.37, \\
142.12,140.84,137.53,136.06,135.70, \\
135.69,134.92,133.44,132.89,130.84, \\
130.48,130.34,129.38,129.14,128.95, \\
128.51,127.83,127.79,127.48,127.33, \\
117.32,48.32,37.87,21.21,10.13 .\end{array}$ & $\begin{array}{l}630.1047 \\
630.1050\end{array}$ \\
\hline $7 \mathbf{k}$ & $\begin{array}{l}\left(400 \mathrm{MHz}, \mathrm{CDCl}_{3}\right) \delta 7.38(\mathrm{~d}, J=1.8 \mathrm{~Hz}, 1 \mathrm{H}, \\
\mathrm{Ar}-\mathrm{H}), 7.31(\mathrm{dd}, J=8.0,1.5 \mathrm{~Hz}, 2 \mathrm{H}, \mathrm{Ar}-\mathrm{H}), \\
7.27-7.10(\mathrm{~m}, 7 \mathrm{H}, \mathrm{Ar}-\mathrm{H}), 7.09-6.98(\mathrm{~m}, 5 \mathrm{H}, \\
\mathrm{Ar}-\mathrm{H}), 6.92-6.84(\mathrm{~m}, 2 \mathrm{H}, \mathrm{Ar}-\mathrm{H}), 4.43(\mathrm{~s}, 2 \mathrm{H}, \\
\left.\mathrm{SCH}_{2}\right), 4.34\left(\mathrm{t}, J=7.6 \mathrm{~Hz}, 2 \mathrm{H}, \mathrm{NC}_{2} \mathrm{CH}_{2}\right), 2.81 \\
\left(\mathrm{t}, J=7.6 \mathrm{~Hz}, 2 \mathrm{H}, \mathrm{NCH}_{2} \mathrm{CH}_{2}\right), 2.29(\mathrm{~s}, 3 \mathrm{H}, \\
\left.\mathrm{CH}_{3}\right) .\end{array}$ & $\begin{array}{l}\left(101 \mathrm{MHz}, \mathrm{CDCl}_{3}\right) \delta 151.01,149.27, \\
142.16,140.86,137.42,136.82,136.22, \\
135.79,134.93,133.03,130.81,130.58, \\
130.36,129.19,128.99,128.91,128.72, \\
128.43,127.90,127.81,127.37,126.70, \\
117.17,46.63,38.00,35.96,10.15 .\end{array}$ & $\begin{array}{l}630.1047 \\
630.1033\end{array}$ \\
\hline 71 & $\begin{array}{l}\left(400 \mathrm{MHz}, \mathrm{CDCl}_{3}\right) \delta 7.39(\mathrm{~d}, J=1.9 \mathrm{~Hz}, 1 \mathrm{H}, \\
\mathrm{Ar}-\mathrm{H}), 7.28-7.09(\mathrm{~m}, 9 \mathrm{H}, \mathrm{Ar}-\mathrm{H}), 7.09-6.98(\mathrm{~m}, \\
5 \mathrm{H}, \mathrm{Ar}-\mathrm{H}), 6.95-6.92(\mathrm{~m}, 2 \mathrm{H}, \mathrm{Ar}-\mathrm{H}), 4.43(\mathrm{t}, J \\
\left.=7.6 \mathrm{~Hz}, 2 \mathrm{H}, \mathrm{NC}_{\underline{H}_{2}} \mathrm{CH}_{2}\right), 3.45(\mathrm{t}, J=7.5 \mathrm{~Hz} \\
\left.2 \mathrm{H}, \mathrm{SC}_{2} \mathrm{CH}_{2}\right), 3.05(\mathrm{t}, J=7.6 \mathrm{~Hz}, 2 \mathrm{H}, \\
\left.\mathrm{NCH}_{2} \mathrm{C}_{2}\right), 2.93\left(\mathrm{t}, J=7.5 \mathrm{~Hz}, 2 \mathrm{H}, \mathrm{SCH}_{2} \underline{\mathrm{CH}}_{2}\right) \\
2.30\left(\mathrm{~s}, 3 \mathrm{H}, \mathrm{CH}_{3}\right) .\end{array}$ & $\begin{array}{l}\left(101 \mathrm{MHz}, \mathrm{CDCl}_{3}\right) \delta 151.35,149.25, \\
142.15,140.93,139.68,137.46,136.25, \\
135.78,134.92,133.05,130.82,130.59, \\
130.36,128.98,128.95,128.81,128.53, \\
128.46,127.90,127.39,126.72,126.59, \\
117.12,46.68,36.01,35.99,34.29,10.17 .\end{array}$ & $\begin{array}{l}644.1204 \\
644.1194\end{array}$ \\
\hline $7 m$ & $\begin{array}{l}\left(400 \mathrm{MHz}, \mathrm{CDCl}_{3}\right) \delta 7.38(\mathrm{~d}, J=2.2 \mathrm{~Hz}, 1 \mathrm{H}, \\
\mathrm{Ar}-\mathrm{H}), 7.26-6.99(\mathrm{~m}, 14 \mathrm{H}, \mathrm{Ar}-\mathrm{H}), 6.96-6.93 \\
(\mathrm{~m}, 2 \mathrm{H}, \mathrm{Ar}-\mathrm{H}), 4.45(\mathrm{t}, J=7.5 \mathrm{~Hz}, 2 \mathrm{H}, \\
\left.\mathrm{NC}_{2} \mathrm{CH}_{2}\right), 3.20(\mathrm{t}, J=7.6 \mathrm{~Hz}, 2 \mathrm{H}, \\
\left.\mathrm{SC}_{2} \mathrm{CH}_{2} \mathrm{CH}_{2}\right), 2.95(\mathrm{t}, J=7.5 \mathrm{~Hz}, 2 \mathrm{H}, \\
\left.\mathrm{NCH}_{2} \mathrm{CH}_{2}\right), 2.69(\mathrm{t}, J=7.6 \mathrm{~Hz}, 2 \mathrm{H}, \\
\left.\mathrm{SCH}_{2} \mathrm{CH}_{2} \underline{\mathrm{C}}_{2}\right), 2.28\left(\mathrm{~s}, 3 \mathrm{H}, \mathrm{CH}_{3}\right), 2.05(\mathrm{p}, J= \\
\left.7.6 \mathrm{~Hz}, 2 \mathrm{H}, \mathrm{SCH}_{2} \underline{\mathrm{C}}_{2} \mathrm{CH}_{2}\right) .\end{array}$ & $\begin{array}{l}\left(101 \mathrm{MHz}, \mathrm{CDCl}_{3}\right) \delta 151.41,149.20, \\
142.12,141.02,140.93,137.45,136.23, \\
135.73,134.88,133.02,130.79,130.57, \\
130.33,128.95,128.93,128.52,128.45, \\
127.87,127.37,126.71,126.03,117.10, \\
46.66,36.01,34.68,32.48,31.17,10.13 .\end{array}$ & $\begin{array}{l}658.1360 \\
658.1348\end{array}$ \\
\hline $7 n$ & $\begin{array}{l}\left(400 \mathrm{MHz}, \mathrm{CDCl}_{3}\right) \delta 7.40(\mathrm{~d}, J=1.8 \mathrm{~Hz}, 1 \mathrm{H}, \\
\mathrm{Ar}-\mathrm{H}), 7.29-7.18(\mathrm{~m}, 5 \mathrm{H}, \mathrm{Ar}-\mathrm{H}), 7.14(\mathrm{~d}, J= \\
8.5 \mathrm{~Hz}, 1 \mathrm{H}, \mathrm{Ar}-\mathrm{H}), 7.10-6.99(\mathrm{~m}, 5 \mathrm{H}, \mathrm{Ar}-\mathrm{H}), \\
6.91-6.88(\mathrm{~m}, 2 \mathrm{H}, \mathrm{Ar}-\mathrm{H}), 6.74(\mathrm{dd}, J=8.5,1.5 \\
\mathrm{Hz}, 2 \mathrm{H}, \mathrm{Ar}-\mathrm{H}), 4.41\left(\mathrm{~s}, 2 \mathrm{H}, \mathrm{SCH}_{2}\right), 4.36(\mathrm{t}, J= \\
\left.7.6 \mathrm{~Hz}, 2 \mathrm{H}, \mathrm{NC}_{2} \mathrm{CH}_{2}\right), 3.67\left(\mathrm{~s}, 3 \mathrm{H}, \mathrm{OCH}_{3}\right), \\
2.84\left(\mathrm{t}, J=7.6 \mathrm{~Hz}, 2 \mathrm{H}, \mathrm{NCH}_{2} \underline{\mathrm{C}}_{2}\right), 2.29(\mathrm{~s}, 3 \mathrm{H}, \\
\left.\mathrm{CH}_{3}\right) .\end{array}$ & $\begin{array}{l}\left(101 \mathrm{MHz}, \mathrm{CDCl}_{3}\right) \delta 159.29,151.22, \\
149.21,142.23,140.83,137.44,136.26, \\
135.86,135.00,133.08,130.85,130.62, \\
130.48,130.42,129.05,128.96,128.71, \\
128.49,127.94,127.40,126.76,117.25, \\
114.17,55.36,46.70,37.67,36.01,10.17 .\end{array}$ & $\begin{array}{l}660.1153 \\
660.1139\end{array}$ \\
\hline 70 & $\begin{array}{l}\left(400 \mathrm{MHz}, \mathrm{CDCl}_{3}\right) \delta 7.40(\mathrm{~d}, J=1.9 \mathrm{~Hz}, 1 \mathrm{H}, \\
\mathrm{Ar}-\mathrm{H}), 7.29-7.18(\mathrm{~m}, 5 \mathrm{H}, \mathrm{Ar}-\mathrm{H}), 7.14(\mathrm{dd}, J= \\
8.5,1.5 \mathrm{~Hz}, 1 \mathrm{H}, \mathrm{Ar}-\mathrm{H}), 7.11-6.98(\mathrm{~m}, 7 \mathrm{H}, \mathrm{Ar}- \\
\mathrm{H}), 6.91-6.88(\mathrm{~m}, 2 \mathrm{H}, \mathrm{Ar}-\mathrm{H}), 4.43(\mathrm{~s}, 2 \mathrm{H}, \\
\left.\mathrm{SCH}_{2}\right), 4.37\left(\mathrm{t}, J=7.6 \mathrm{~Hz}, 2 \mathrm{H}, \mathrm{NC}_{2} \mathrm{CH}_{2}\right), 2.84 \\
\left(\mathrm{t}, J=7.6 \mathrm{~Hz}, 2 \mathrm{H}, \mathrm{NCH}_{2} \mathrm{CH}_{2}\right), 2.29(\mathrm{~s}, 3 \mathrm{H}, \\
\left.\mathrm{CH}_{3}\right), 2.22\left(\mathrm{~s}, 3 \mathrm{H}, \mathrm{CH}_{3}\right) .\end{array}$ & $\begin{array}{l}\left(101 \mathrm{MHz}, \mathrm{CDCl}_{3}\right) \delta 151.23,149.22, \\
142.21,140.86,137.64,137.45,136.26, \\
135.84,134.99,133.64,133.08,130.85, \\
130.61,130.41,129.46,129.17,129.04, \\
128.96,128.48,127.94,127.40,126.75 \\
117.24,46.71,37.78,35.99,21.24,10.17 .\end{array}$ & $\begin{array}{l}644.1204 \\
644.1193\end{array}$ \\
\hline
\end{tabular}




\section{Anticancer screening in National Cancer Institute (NCI)}

The methodology of the NCI anticancer screening has been described in detail elsewhere (http://www.dtp.nci.nih.gov) ${ }^{46-48}$. Briefly, the primary anticancer assay was performed at approximately 60 human tumor cell lines panel derived from nine neoplastic diseases in accordance with the protocol of the Drug Evaluation Branch, National Cancer Institute, reported elsewhere. Tested compounds were added to the culture at a single concentration $\left(10^{-5} \mathrm{M}\right)$ and the cultures were incubated for $48 \mathrm{~h}$. End point determinations were made with a protein binding dye, SRB. Results for each tested compound were reported as the percent of growth of the treated cells when compared to the untreated control cells. The percentage growth was evaluated spectrophotometrically versus controls not treated with test agents. The cytotoxic and/or growth inhibitory effects of the most active selected compound were tested in-vitro against the full panel of about 60 human tumor cell lines at 10 -fold dilutions of five concentrations ranging from $10^{-4}$ to $10^{-8} \mathrm{M}$. A 48-h continuous drug exposure protocol was followed and an SRB protein assay was used to estimate cell viability or growth. Using the seven absorbance measurements [time zero (Tz), control growth in the absence of drug $(\mathrm{C})$, and test growth in the presence of drug at the five concentration levels (Ti)], the percentage growth was calculated at each of the drug concentrations levels. Percentage growth inhibition was calculated as: $[(\mathrm{Ti}-\mathrm{Tz}) /(\mathrm{C}-$ $\mathrm{Tz})] \times 100$ for concentrations for which $\mathrm{Ti} \geq$ $\mathrm{Tz}$, and $[(\mathrm{Ti}-\mathrm{Tz}) / \mathrm{Tz}] \times 100$ for concentrations for which $\mathrm{Ti}<\mathrm{Tz}$. Three-dose response parameters $\left(\mathrm{GI}_{50}, \mathrm{TGI}\right.$, and $\left.\mathrm{LC}_{50}\right)$ were calculated for each compound. Growth inhibition of $50 \%\left(\mathrm{GI}_{50}\right)$ was calculated from $[(\mathrm{Ti}-\mathrm{Tz}) /(\mathrm{C}-\mathrm{Tz})] \times 100=50$, which is the drug concentration resulting in a 50\% lower net protein increase in the treated cells (measured by SRB staining) as compared to the net protein increase seen in the control cells. The drug concentration resulting in total growth inhibition (TGI) was calculated from $\mathrm{Ti}=\mathrm{Tz}$. The $\mathrm{LC}_{50}$ (concentration of drug resulting in a $50 \%$ reduction in the measured protein at the end of the drug treatment as compared to that at the beginning) indicating a net loss of cells following treatment was calculated from [(Ti $-\mathrm{Tz}) / \mathrm{Tz}] \times 100=-50$. Values were calculated for each of these three parameters if the level of activity is reached; however, if the effect was not reached or was exceeded, the value for that parameter was expressed as more or less than the maximum or minimum concentration tested. The $\log \mathrm{GI}_{50}, \log \mathrm{TGI}$, and $\operatorname{logLC} \mathrm{C}_{50}$ were then determined. $\log \mathrm{GI}_{50}, \log \mathrm{TGI}$, and $\log$ $\mathrm{LC}_{50}$ are the logarithm molar concentrations producing $50 \%$ growth inhibition $\left(\mathrm{GI}_{50}\right)$, a total growth inhibition (TGI), and a 50\% cellular death $\left(\mathrm{LC}_{50}\right)$, respectively. The lowest values are obtained with the most sensitive cell lines.

\section{Anticancer screening in National Research Centre, Egypt}

Chemicals: Fetal bovine serum (FBS) and Lglutamine, were obtained from Gibco Invitrogen Company (Scotland, UK). Dulbecco's modified Eagle's (DMEM) medium was provided from Cambrex (New Jersey, USA). Dimethyl sulfoxide (DMSO), doxorubicin, penicillin, streptomycin and Sulfo-Rhodamine-B stain (SRB) (3-(4,5dimethylthiazol-2-yl)-2,5-diphenyltetrazolium bromide) were obtained from Sigma-Aldrich Chemical Company (St. Louis, MO, USA). All other chemicals and reagents used in this study were of analytical grade and purchased from Sigma-Aldrich Chemical Co. (St. Louis, MO, USA).

Cell lines and culturing: Anticancer activity screening for the tested compounds breast MCF-7, lung A549 cancer cell lines as well as the normal cell line (human normal melanocyte, HFB4) were obtained from the American Type Culture Collection (Rockville, MD, USA). The cells were maintained in Dulbecco's modified Eagle's medium (DMEM) supplemented with $10 \%$ heat inactivated fetal calf serum (GIBCO), penicillin $(100 \mathrm{U} / \mathrm{mL})$ and streptomycin $(100 \mu \mathrm{g} / \mathrm{mL})$ at $37{ }^{\circ} \mathrm{C}$ in humidified atmosphere containing $5 \% \quad \mathrm{CO}_{2}$. Cells at a concentration of $0.50 \times 10^{6}$ were grown in a $25 \mathrm{~cm}^{2}$ flask in $5 \mathrm{~mL}$ of complete culture medium.

In-vitro cytotoxicity assay: The cytotoxicity activity was measured in-vitro using the Sulforhodamine-B stain (SRB) assay according to the previous reported standard procedure ${ }^{49}$. 
Cells were inoculated in 96-well microtiter plate $\left(10^{4}\right.$ cells/ well) for $24 \mathrm{~h}$ before treatment with the tested compounds to allow attachment of cell to the wall of the plate. The tested compounds were dissolved in DMSO at 1 $\mathrm{mg} / \mathrm{mL}$ immediately before use and diluted to the appropriate volume just before addition to the cell culture. Different concentration of tested compounds and doxorubicin were added to the cells. Triplicate wells were prepared for each individual dose. Cells were incubated with the compounds for $48 \mathrm{~h}$ at $37^{\circ} \mathrm{C}$ and in atmosphere of $5 \% \mathrm{CO}_{2}$. After $48 \mathrm{~h}$ cells were fixed, washed, and stained for $30 \mathrm{~min}$ with $0.4 \%(\mathrm{w} / \mathrm{v})$ SRB dissolved in $1 \%$ acetic acid. Unbound dye was removed by four washes with $1 \%$ acetic acid, and attached stain was recovered with Tris-EDTA buffer. Colour intensity was measured in an ELISA reader. The relation between surviving fraction and drug concentration is plotted to get the survival curve for each cell line after the specified time. The concentration required for $50 \%$ inhibition of cell viability $\left(\mathrm{IC}_{50}\right)$ was calculated and the results are given in table III. The results were compared to the antiproliferative effects of the reference control doxorubicin.

\section{RESULTS AND DISCUSSION}

\section{1- Chemistry}

The synthetic route of the designated compounds was shown in scheme 1. 5-(4chlorophenyl)-1-(2,4-dichlorophenyl)-4methyl-1H-pyrazole-3-carboxylate $\mathbf{3}$ was synthesised through treatment of 1-(4chlorophenyl)propanone 1 with diethyl oxalate in the presence of LHMDS as a base to afford lithium salt 2 in $80 \%$ yield, which in turn was coupled with 2,4-dichlorophenylhydrazine hydrochloride in ethanol followed by intramolecular cyclization in acetic acid under refluxing conditions to provide the pyrazole-3carboxylic acid ethyl ester 3 in 55\% yield over two steps ${ }^{50}$. The ${ }^{1} \mathrm{H}-\mathrm{NMR}$ spectrum of $\mathbf{3}$ showed a singlet equivalent to three protons at $\delta 2.31 \mathrm{ppm}$ which assigned to methyl groupand a quartet at $4.42 \mathrm{ppm}$ and a triplet at $1.39 \mathrm{ppm}$ related to ethoxy moiety as well as aromatic protons appeared at expected chemical shift. The key hydrazide intermediate $\mathbf{4}$ was prepared in a high yield by reflux pyrazole-3-carboxylic acid ester 3 with hydrazine in ethanol. The structure of compound $\mathbf{4}$ was confirmed by NMR and ESI-HRMS. Heating at reflux a mixture of the hydrazide $\mathbf{4}$ and an appropriate isothiocyanate in ethanol afforded substituted thiosemicarbazides 5a-c which was used for next step without further purification. A solution of 5a-c in $2 \mathrm{~N} \mathrm{NaOH}$ was stirred under reflux for $3 \mathrm{~h}$ to yield 1,2,4-triazole-3-thiol derivatives 6a-c. All of the synthetic compounds of this series gave satisfactory spectroscopic and HRESI-MS data, which were in full accordance with their depicted structures. The ${ }^{1} \mathrm{H}$ NMR spectrum of $\mathbf{6 a}$ as a representative example of this series revealed in addition to aromatic proton the appearance of ethyl group signals at $\delta 4.36,1.21 \mathrm{ppm}$. The structure of $\mathbf{6 a}$ has been also confirmed by HRESI-MS data. The synthesis of the final compounds 7a-o was accomplished by refluxing 1,2,4-triazole-3-thiols 6a-c with benzyl bromide, phenethyl bromide, 3phenylpropyl bromide, 4-methoxybenzyl chloride, or 4-methylbenzyl bromide in the presence of $\mathrm{K}_{2} \mathrm{CO}_{3}$ in acetone. All the final structures 7a-o were verified using ${ }^{1} \mathrm{H}$ NMR, ${ }^{13} \mathrm{C}$ NMR and HRESI-MS. Analysis of the ${ }^{1} \mathrm{H}$ NMR spectrum of $\mathbf{7 a}$ as an example of this series showed the appearance of a signal at $4.43 \mathrm{ppm}$ assigned to $\mathrm{SCH}_{2}$ group, ethyl protons at 4.17 (q) and $1.12(\mathrm{t}) \mathrm{ppm}$ and a singlet signal integrating for three protons at $2.33 \mathrm{ppm}$ which was attributed to methyl group as well as aromatic protons at the expected chemical shift. Additional confirmation of the structure of 7a was provided by HRESI-MS data, which showed a peak at $\mathrm{m} / \mathrm{z} 554.0721$ for $[\mathrm{M}+\mathrm{H}]^{+}$, which consistent with the molecular formula $\mathrm{C}_{27} \mathrm{H}_{23} \mathrm{Cl}_{3} \mathrm{~N}_{5} \mathrm{~S}$.

\section{2- Screening of anticancer activity by NCI}

\section{In-vitro one-dose full NCI 60 cell panel assay}

Compounds $\mathbf{7 a}, \mathbf{7 b}, \mathbf{7 d}-\mathbf{g}$ were selected by the National Cancer Institute (NCI) according to the protocol of the Drug Evaluation Branch of the National Cancer Institute, Bethesda, USA for invitro anticancer screening ${ }^{46}$. Primary in-vitro onedose anticancer assay was performed in full NCI 60 cell lines derived from nine tumor subpanels, including leukemia, melanoma, lung, colon, CNS, ovarian, renal, prostate, and breast cancer cell lines. The selected compounds were added at a single concentration $\left(10^{-5} \mathrm{M}\right)$ and the culture was incubated for $48 \mathrm{~h}$. End point determination was 
made with a protein binding dye sulforhodamine B (SRB). Results for each compound were reported as a mean graph of the percent growth of the treated cells when compared to the untreated control cells.

Compound 7e achieved remarkable cell growth inhibition activity against most of the tested cell lines (Table III). A complete cell death was recorded for leukemia HL-60(TB), RPMI8226, non-small cell lung cancer HOP-92, NCIH522, colon cancer COLO 205, melanoma, SKMEL-5, UACC-257, ovarian cancer SK-OV-3, breast cancer BT-549, T-47D cell lines. Compound 7e indicated a remarkable cell growth inhibition activity against most of the tested cell lines including leukemia K-562, MOLT-4, SR, non-small cell lung cancer A549/ATCC, EKVX, HOP-62, NCI-H226, NCIH23, NCI-H460, colon cancer HCC-2998, HCT116, НCT-15, HT29, KM12, SW-620, CNS cancer SF-268, SF-295, SF-539, SNB-19, SNB75, U251, melanoma LOX IMVI, MALME-3M, M14, MDA-MB-435, SK-MEL-2, UACC-62, ovarian cancer OVCAR-3, OVCAR-4, OVCAR5, OVCAR-8, renal cancer 786-0, A498, ACHN, RXF 393, SN12C, TK-10, UO-31, prostate cancer PC-3, DU-145, breast cancer MCF7, MDA-MB-231/ATCC, MDA-MB-468 cell lines. Compound 7e revealed moderate cell growth inhibition against melanoma SK-MEL-28, ovarian cancer IGROV1, renal cancer CAKI-1, breast cancer HS 578T cell lines. The results also indicated that $\mathbf{7 a}$ revealed a remarkable cell growth inhibition activity only against prostate cancer PC-3 cell line and exhibited moderate cell growth inhibition against non-small cell lung cancer EKVX, colon cancer HCT-116, HCT-15, CNS cancer SF-295, renal cancer ACHN, UO-31, breast cancer MCF7, T-47D, MDA-MB-468 cell lines. The obtained results indicate that compound 7e exhibited the highest ability to inhibit the proliferation of different cancer cell lines (Table III) compared to compounds $\mathbf{7 a}, \mathbf{7 b}$, 7d, 7f-g.

From the obtained results; several conclusions could be deduced, a 1,5diarylpyrazole functionality attached to the 1,2,4triazole moiety might contribute to the anticancer activity of the synthesized compounds. It could be noted that compound 7e with weak electron donating groups $\left(\mathrm{CH}_{3}\right)$ in 4-position of benzyl moiety at 5-position of 1,2,4-triazole is more active than compound $\mathbf{7 d}$ with strong electron donating groups $\left(\mathrm{OCH}_{3}\right)$ Moreover, the presence of ethyl at 4-position of 1,2,4-triazole is preferable over the presence of benzyl group (compound 7e has superior anticancer activity against different cancer cell lines over all tested compounds by NCI).

Table III: One-dose growth (\%) of nine different cancer cell types for compounds 7a, 7b, 7d-g.

\begin{tabular}{|c|c|c|c|c|c|c|}
\hline \multirow{2}{*}{ Panel/cell line } & \multicolumn{6}{|c|}{ Growth $(\%)$ in one-dose assay } \\
\hline & $7 a$ & $7 b$ & 7d & $7 e$ & $7 f$ & $7 g$ \\
\hline \multicolumn{7}{|l|}{ Leukemia } \\
\hline CCRF-CEM & 81.95 & NT & 85.38 & NT & NT & NT \\
\hline HL-60(TB) & 61.45 & 67.83 & 76.73 & -13.10 & 62.12 & 82.38 \\
\hline K-562 & 53.97 & 48.11 & 77.68 & 8.88 & 49.93 & 59.13 \\
\hline MOLT-4 & 51.41 & 41.59 & 79.22 & 2.46 & 39.89 & 64.63 \\
\hline RPMI-8226 & 56.85 & 54.07 & 60.90 & -3.78 & 51.43 & 74.78 \\
\hline SR & 57.72 & 37.63 & 77.13 & 6.14 & 39.72 & 46.40 \\
\hline \multicolumn{7}{|c|}{$\begin{array}{l}\begin{array}{l}\text { Non-Small } \\
\text { Cancer }\end{array} \\
\end{array}$} \\
\hline A549/ATCC & 56.12 & 65.06 & 87.08 & 9.87 & 56.19 & 69.23 \\
\hline EKVX & 42.68 & 52.56 & 58.03 & 10.41 & 52.60 & 69.65 \\
\hline HOP-62 & 85.07 & 101.43 & 91.93 & 9.33 & 80.67 & 87.53 \\
\hline HOP-92 & 57.34 & 84.96 & 85.40 & -17.19 & 67.18 & 96.46 \\
\hline NCI-H226 & 59.60 & 57.99 & 68.27 & 11.63 & 64.49 & 62.80 \\
\hline NCI-H23 & 51.90 & 62.25 & 64.87 & 3.53 & 59.70 & 72.66 \\
\hline NCI-H322M & 76.44 & NT & 94.02 & NT & NT & NT \\
\hline NCI-H460 & 64.44 & 73.73 & 75.16 & 8.69 & 68.08 & 78.60 \\
\hline NCI-H522 & 62.21 & 61.12 & 64.07 & -17.45 & 53.74 & 61.33 \\
\hline \multicolumn{7}{|l|}{ Colon Cancer } \\
\hline COLO 205 & 77.57 & 77.72 & 84.48 & -34.35 & 77.74 & 83.34 \\
\hline HCC-2998 & 86.09 & 110.06 & 94.82 & 23.36 & 79.38 & 97.90 \\
\hline НCT-116 & 40.34 & 39.08 & 47.27 & 1.74 & 47.55 & 37.04 \\
\hline
\end{tabular}




\begin{tabular}{|c|c|c|c|c|c|c|}
\hline \multirow{2}{*}{$\begin{array}{l}\text { Table fil: Continued. } \\
\text { Panel/cell line }\end{array}$} & \multicolumn{6}{|c|}{ Growth (\%) in one-dose assay } \\
\hline & $7 \mathbf{a}$ & $7 \mathbf{b}$ & $7 d$ & $7 \mathrm{e}$ & $7 f$ & $7 \mathrm{~g}$ \\
\hline HCT-15 & 45.07 & 45.62 & 74.04 & 11.58 & 59.79 & 56.57 \\
\hline HT29 & 73.56 & 67.15 & 67.37 & 2.65 & 49.53 & 75.18 \\
\hline KM12 & 56.67 & 69.54 & 66.39 & 15.84 & 63.02 & 77.81 \\
\hline SW-620 & 71.46 & 83.34 & 77.33 & 20.37 & 72.94 & 83.80 \\
\hline \multicolumn{7}{|l|}{ CNS Cancer } \\
\hline SF-268 & 59.11 & 77.35 & 83.61 & 22.84 & 75.89 & 76.91 \\
\hline SF-295 & 45.48 & 65.75 & 83.04 & 9.65 & 61.23 & 82.90 \\
\hline SF-539 & 90.97 & 85.86 & 98.20 & 19.78 & 82.34 & 83.76 \\
\hline SNB-19 & 75.41 & 76.75 & 87.25 & 22.07 & 78.83 & 67.98 \\
\hline SNB-75 & 71.33 & 90.68 & 85.07 & 23.30 & 67.79 & 89.17 \\
\hline U251 & 65.72 & 54.74 & 87.72 & 14.44 & 66.42 & 52.20 \\
\hline \multicolumn{7}{|l|}{ Melanoma } \\
\hline LOX IMVI & 56.79 & 63.77 & 75.35 & 7.19 & 60.05 & 60.72 \\
\hline MALME-3M & 76.86 & 95.99 & 84.80 & 7.80 & 87.94 & 91.77 \\
\hline M14 & 62.22 & 72.78 & 80.62 & 12.27 & 71.58 & 82.08 \\
\hline MDA-MB-435 & 71.41 & 83.18 & 91.13 & 19.69 & 80.64 & 90.01 \\
\hline SK-MEL-2 & 66.11 & 53.80 & 76.22 & 4.93 & 65.79 & 68.05 \\
\hline SK-MEL-28 & 85.86 & 79.20 & 96.85 & 30.32 & 86.82 & 87.58 \\
\hline SK-MEL-5 & 57.73 & 74.26 & 66.51 & -92.32 & 73.08 & 72.65 \\
\hline UACC-257 & 85.19 & 77.84 & 98.23 & -1.54 & 72.19 & 75.98 \\
\hline UACC-62 & 60.77 & 68.63 & 78.80 & 21.19 & 57.52 & 68.37 \\
\hline \multicolumn{7}{|l|}{ Ovarian Cancer } \\
\hline IGROV1 & 84.42 & 94.08 & 97.46 & 30.85 & 92.03 & 87.56 \\
\hline OVCAR-3 & 56.55 & 69.06 & 65.91 & 15.82 & 68.30 & 70.94 \\
\hline OVCAR-4 & 53.20 & 75.61 & 66.43 & 16.89 & 56.54 & 68.70 \\
\hline OVCAR-5 & 81.13 & 81.72 & 96.02 & 26.76 & 88.64 & 87.41 \\
\hline OVCAR-8 & 76.89 & 84.55 & 95.86 & 12.85 & 83.56 & 91.25 \\
\hline NCI/ADR-RES & 64.57 & 72.64 & 87.65 & 52.12 & 77.40 & 91.21 \\
\hline SK-OV-3 & 86.05 & 79.07 & 86.39 & -7.13 & 75.46 & NT \\
\hline \multicolumn{7}{|l|}{ Renal Cancer } \\
\hline $786-0$ & 73.38 & 72.89 & 84.09 & 12.17 & 74.07 & 79.51 \\
\hline A498 & 85.98 & 96.56 & 83.13 & 18.85 & 95.57 & 95.24 \\
\hline $\mathrm{ACHN}$ & 46.12 & 60.98 & 80.48 & 2.32 & 65.48 & 76.66 \\
\hline CAKI-1 & 57.13 & 64.39 & 69.94 & 40.52 & 56.85 & 74.01 \\
\hline RXF 393 & 80.08 & 66.04 & 90.81 & 8.71 & 84.66 & 83.26 \\
\hline SN12C & 50.22 & 70.33 & 90.92 & 1.66 & 67.90 & 86.09 \\
\hline TK-10 & 84.75 & 75.30 & 81.96 & 18.10 & 75.58 & 74.53 \\
\hline UO-31 & 43.91 & 53.53 & 79.11 & 9.59 & 60.23 & 72.72 \\
\hline \multicolumn{7}{|l|}{ Prostate Cancer } \\
\hline PC-3 & 25.66 & 39.80 & 48.82 & 5.63 & 40.69 & 49.39 \\
\hline DU-145 & 79.10 & 84.67 & 90.63 & 23.38 & 85.00 & 92.86 \\
\hline \multicolumn{7}{|l|}{ Breast Cancer } \\
\hline MCF7 & 43.89 & 58.70 & 62.67 & 4.46 & 57.33 & 71.96 \\
\hline MDA-MB-231/ATCC & 71.62 & 81.06 & 73.01 & 5.05 & 71.21 & 78.19 \\
\hline HS 578T & 76.73 & 97.95 & 91.56 & 38.41 & 91.19 & 92.62 \\
\hline BT-549 & 83.19 & 93.35 & 91.65 & -7.34 & 81.77 & 100.14 \\
\hline $\mathrm{T}-47 \mathrm{D}$ & 35.76 & 42.20 & 61.89 & -8.15 & 41.25 & 51.29 \\
\hline MDA-MB-468 & 45.14 & 45.88 & 62.48 & 6.59 & 58.89 & 68.15 \\
\hline Mean & 64.77 & 70.37 & 79.50 & 8.80 & 67.82 & 75.98 \\
\hline Range & 65.31 & 72.43 & 50.96 & 144.44 & 55.85 & 63.10 \\
\hline
\end{tabular}

NT: not tested.

\section{In-vitro five-dose full NCI 60 cell panel assay}

Compound 7e (NSC: D-785448/1) was satisfied the threshold inhibition criteria and selected for advanced five-dose testing against the full panel of 60 human tumor cell lines. All the 60 cell lines representing nine tumor subpanels were incubated at five different concentrations $(0.01,0.1,1,10$ and $100 \mu \mathrm{M})$. The outcomes were used to create $\log$ concentration versus \% growth inhibition 
curves and three response parameters $\left(\mathrm{GI}_{50}\right.$, TGI, and $\mathrm{LC}_{50}$ ) were calculated for each cell line. The $\mathrm{GI}_{50}$ value (growth inhibitory activity) corresponds to the concentration of the compound causing 50\% decrease in net cell growth, the TGI value (cytostatic activity) is the concentration of the compound resulting in total growth inhibition (TGI) and $\mathrm{LC}_{50}$ value (cytotoxic activity) is the concentration of the compound causing net $50 \%$ loss of initial cells at the end of the incubation period of $48 \mathrm{~h}$. The results in table IV indicated that compound 7e exhibited remarkable anticancer activity against most of the tested cell lines representing nine different subpanels with $\mathrm{GI}_{50}$ ranging from 0.43 to $3.55 \mu \mathrm{M}$.
The criterion for selectivity of a compound depends upon the ratio obtained by dividing the full panel MID (the average sensitivity of all cell lines toward the test agent) $(\mu \mathrm{M})$ by their individual subpanel MID $(\mu \mathrm{M})$. Ratios between 3 and 6 refer to moderate selectivity; ratios $>6$ indicate high selectivity toward the corresponding cell line, while compounds not meeting either of these criteria rated non selective. In this context, compound 7e was found to have broad spectrum antitumor activity against the nine tumour subpanels tested with no selectivity toward the tested cell lines (selectivity ratios ranging between 0.80 and 1.39 at the $\mathrm{GI}_{50}$ level).

Table IV: NCI in-vitro testing results of compound $\mathbf{7 e}$ at five-dose level in mM.

\begin{tabular}{|c|c|c|c|c|c|}
\hline \multirow{2}{*}{ Panel/cell line } & \multicolumn{3}{|c|}{$\mathrm{GI}_{50}$} & \multirow[t]{2}{*}{ TGI } & \multirow[t]{2}{*}{$\mathrm{LC}_{50}$} \\
\hline & $\begin{array}{l}\text { Conc. per cell } \\
\text { line }\end{array}$ & $\begin{array}{l}\text { Subpanel } \\
\text { MID }^{\mathrm{b}}\end{array}$ & Selectivity ratio & & \\
\hline \multicolumn{6}{|l|}{ Leukemia } \\
\hline CCRF-CEM & 2.51 & \multirow{5}{*}{1.51} & \multirow{5}{*}{1.38} & 13.5 & $>100$ \\
\hline HL-60(TB) & 1.60 & & & 5.47 & 35.60 \\
\hline K-562 & 0.84 & & & 10.40 & 44.20 \\
\hline MOLT-4 & 2.15 & & & 11.00 & 46.70 \\
\hline RPMI-8226 & 0.47 & & & 3.05 & 90.20 \\
\hline \multicolumn{6}{|c|}{$\begin{array}{l}\text { Non-Small Cell Lung } \\
\text { Cancer }\end{array}$} \\
\hline A549/ATCC & 2.82 & \multirow{9}{*}{2.11} & \multirow{9}{*}{0.99} & 11.30 & 34.70 \\
\hline EKVX & 2.88 & & & 13.00 & 38.40 \\
\hline HOP-62 & 2.73 & & & 11.90 & 38.10 \\
\hline HOP-92 & 0.53 & & & 4.22 & 30.90 \\
\hline NCI-H226 & 2.26 & & & 13.90 & 51.30 \\
\hline NCI-H23 & 2.24 & & & 12.5 & 42.80 \\
\hline NCI-H322M & 2.71 & & & 13.00 & 36.90 \\
\hline NCI-H460 & 1.46 & & & 10.50 & 38.10 \\
\hline NCI-H522 & 1.35 & & & 5.20 & 23.60 \\
\hline \multicolumn{6}{|l|}{ Colon Cancer } \\
\hline COLO 205 & 1.51 & \multirow{7}{*}{1.69} & \multirow{7}{*}{1.23} & 3.39 & 7.61 \\
\hline HCC-2998 & 2.35 & & & 13.20 & 39.00 \\
\hline HCT-116 & 0.57 & & & 10.60 & 36.30 \\
\hline HCT-15 & 2.70 & & & 11.40 & 35.70 \\
\hline HT29 & 1.11 & & & 10.60 & 34.70 \\
\hline KM12 & 1.04 & & & 6.31 & 31.90 \\
\hline SW-620 & 2.56 & & & 12.60 & 39.30 \\
\hline \multicolumn{6}{|l|}{ CNS Cancer } \\
\hline SF-268 & 3.06 & \multirow{6}{*}{2.32} & \multirow{6}{*}{0.90} & 14.90 & 46.40 \\
\hline SF-295 & 2.40 & & & 11.70 & 35.80 \\
\hline SF-539 & 3.02 & & & 11.80 & 35.50 \\
\hline SNB-19 & 2.30 & & & 13.30 & 38.70 \\
\hline SNB-75 & 2.21 & & & 11.70 & 35.60 \\
\hline $\mathrm{U} 251$ & 0.93 & & & 11.80 & 35.40 \\
\hline \multicolumn{6}{|l|}{ Melanoma } \\
\hline LOX IMVI & 2.72 & & & 10.70 & 34.30 \\
\hline MALME-3M & 2.15 & & & 8.88 & 32.20 \\
\hline M14 & 1.99 & & & 10.80 & 34.80 \\
\hline
\end{tabular}




\begin{tabular}{|c|c|c|c|c|c|}
\hline \multirow[b]{2}{*}{ Table IV: Continued } & \multicolumn{3}{|c|}{$\mathrm{GI}_{50}$} & \multirow[t]{2}{*}{ TGI } & \multirow[t]{2}{*}{$\mathrm{LC}_{50}$} \\
\hline & $\begin{array}{l}\text { Conc. per cell } \\
\text { line }\end{array}$ & $\begin{array}{l}\text { Subpanel } \\
\text { MID }^{\mathrm{b}}\end{array}$ & Selectivity ratio & & \\
\hline MDA-MB-435 & 2.85 & \multirow{6}{*}{2.34} & \multirow[t]{6}{*}{0.89} & 11.50 & 34.80 \\
\hline SK-MEL-2 & 3.01 & & & 12.50 & 37.90 \\
\hline SK-MEL-28 & 3.11 & & & 15.30 & 41.30 \\
\hline SK-MEL-5 & 1.40 & & & 2.74 & 5.38 \\
\hline UACC-257 & 1.86 & & & 4.73 & 15.40 \\
\hline UACC-62 & 2.01 & & & 10.60 & 34.90 \\
\hline \multicolumn{6}{|l|}{ Ovarian Cancer } \\
\hline IGROV1 & 2.62 & \multirow{7}{*}{2.60} & \multirow{7}{*}{0.80} & 15.40 & 42.80 \\
\hline OVCAR-3 & 1.98 & & & 11.60 & 35.70 \\
\hline OVCAR-4 & 1.79 & & & 12.40 & 38.80 \\
\hline OVCAR-5 & 3.55 & & & 15.20 & 40.80 \\
\hline OVCAR-8 & 2.12 & & & 10.70 & 36.60 \\
\hline NCI/ADR-RES & 3.54 & & & 14.60 & 44.30 \\
\hline SK-OV-3 & 2.58 & & & 8.18 & 28.80 \\
\hline \multicolumn{6}{|l|}{ Renal Cancer } \\
\hline $786-0$ & 2.69 & \multirow{8}{*}{2.49} & \multirow{8}{*}{0.84} & 12.20 & 36.40 \\
\hline A498 & 1.37 & & & 7.88 & 30.60 \\
\hline $\mathrm{ACHN}$ & 2.74 & & & 10.40 & 35.50 \\
\hline CAKI-1 & 2.83 & & & 12.60 & 36.50 \\
\hline RXF 393 & 2.69 & & & 10.90 & 36.10 \\
\hline SN12C & 2.31 & & & 10.80 & 37.20 \\
\hline TK-10 & 2.61 & & & 10.90 & 35.30 \\
\hline UO-31 & 2.66 & & & 10.80 & 34.00 \\
\hline \multicolumn{6}{|l|}{ Prostate Cancer } \\
\hline PC-3 & 0.49 & \multirow[t]{2}{*}{1.90} & \multirow[t]{2}{*}{1.09} & 10.40 & 38.50 \\
\hline DU-145 & 3.30 & & & 13.80 & 40.40 \\
\hline \multicolumn{6}{|l|}{ Breast Cancer } \\
\hline MCF7 & 2.26 & \multirow{6}{*}{1.79} & \multirow{6}{*}{1.16} & 11.40 & 47.10 \\
\hline MDA-MB-231/ATCC & 2.35 & & & 8.54 & 32,10 \\
\hline HS 578T & 3.30 & & & 18.40 & 92.10 \\
\hline BT-549 & 1.79 & & & 5.75 & 23.60 \\
\hline T-47D & 0.43 & & & 10.10 & 42.10 \\
\hline MDA-MB-468 & 0.62 & & & 10.60 & 39.20 \\
\hline $\mathrm{MID}^{\mathrm{a}}$ & & 2.08 & & & \\
\hline
\end{tabular}

\section{3- Screening of anticancer activity by National Research Centre, Egypt}

The cytotoxicity of the synthetic final compounds except 7e was tested using SRB assay as described by Skehan $e t a l^{49}$ against breast MCF-7, lung A549 cell lines as well as human normal melanocyte (HFB4) cells using doxorubicin as a reference drug and DMSO as a control (Table V). Moreover, the tumor cells showed normal growth in culture system and DMSO did not seem to have any noticeable effect on cellular growth. The results revealed that compounds $\mathbf{7 l}$ and 70 exhibited higher potency against MCF-7 and A549 cells with $\mathrm{IC}_{50}: 2.85 \pm 0.35$ and $2.84 \pm 0.37 \mu \mathrm{g} / \mathrm{mL}$ against MCF-7 and $\mathrm{IC}_{50}$ : $4.11 \pm 0.50$ and $3.90 \pm 0.48$ $\mu \mathrm{g} / \mathrm{mL}$ against A549 cells, respectively, which is lower than of doxorubicin as shown in table III. Moreover, the results showed that compounds $\mathbf{7 m}$ and $\mathbf{7 n}$ were found to be equipotent to doxorubicin against MCF-7 cells with $\mathrm{IC}_{50}: 3.70 \pm 4.00$ and $3.30 \pm 0.36 \mu \mathrm{g} / \mathrm{ml}$ and against $\mathrm{A} 549$ cells with $\mathrm{IC}_{50}: 8.86 \pm 0.90$ and $6.86 \pm 0.87 \mu \mathrm{g} / \mathrm{mL}$, respectively. The rest of compounds revealed moderate to low anticancer effect as shown in table $\mathrm{V}$. Additionally, while compounds 7c-d, 7l-o exhibited no activity against the growth of normal HFB4 cells, the rest compounds revealed high toxicity on the normal cells.

From the above mentioned results, it is clear that in general, biological activity against cancer cells depends on the presence of 1,5diarylpyrazole nucleus and the chemical nature of the substituents R, R1 at triazole moiety. The compounds 7l-o with phenethyl group at 4position of 1,2,4-triazole were highly active against two cancer cell lines (MCF-7 and 
A549). These results also confirmed that the presence of either benzyl group with electron donating groups $\left(\mathrm{CH}_{3}\right.$ and $\left.\mathrm{OCH}_{3}\right)$ at its $p$ - position or phenethyl group is preferable over unsubstituted benzyl group for anticancer activity.

Table V: In-vitro cytotoxicity activity of the tested compounds $7 \mathbf{a}-\mathbf{d}, \mathbf{7 f}-\mathbf{o}$ on different cell lines.

\begin{tabular}{|c|c|c|c|}
\hline \multirow{2}{*}{ Compd. } & \multicolumn{3}{|c|}{ IC $_{\mathbf{5 0}}(\boldsymbol{\mu} \mathbf{g} / \mathbf{m L})$} \\
\cline { 2 - 4 } & MCF-7 & A549 & HFB4 \\
\hline $\mathbf{7 a}$ & N.A. & N.A. & $8.03 \pm 0.80$ \\
\hline $\mathbf{7 b}$ & N.A. & N.A. & $9.76 \pm 0.74$ \\
\hline $\mathbf{7 c}$ & N.A. & N.A. & N.A. \\
\hline $\mathbf{7 d}$ & N.A. & N.A. & N.A. \\
\hline $\mathbf{7 f}$ & $23.90 \pm 3.31$ & $62.30 \pm 7.11$ & $4.63 \pm 0.50$ \\
\hline $\mathbf{7 g}$ & $13.80 \pm 2.50$ & $21.31 \pm 2.60$ & $40.73 \pm 5.20$ \\
\hline $\mathbf{7 h}$ & $26.20 \pm 3.40$ & $41.11 \pm 4.26$ & $6.71 \pm 0.75$ \\
\hline $\mathbf{7 i}$ & $18.56 \pm 2.20$ & $16.96 \pm 2.46$ & $8.11 \pm 0.73$ \\
\hline $\mathbf{7 j}$ & $36.80 \pm 3.96$ & $23.29 \pm 2.94$ & $7.62 \pm 0.85$ \\
\hline $\mathbf{7 k}$ & $19.27 \pm 2.11$ & $19.80 \pm 2.48$ & $27.53 \pm 2.92$ \\
\hline $\mathbf{7 l}$ & $2.84 \pm 0.37$ & $3.90 \pm 0.48$ & $80.13 \pm 9.00$ \\
\hline $\mathbf{7 m}$ & $3.70 \pm 4.00$ & $8.86 \pm 0.90$ & $66.89 \pm 7.00$ \\
\hline $\mathbf{7 n}$ & $3.30 \pm 0.36$ & $6.86 \pm 0.87$ & $65.94 \pm 8.77$ \\
\hline $\mathbf{7 0}$ & $2.85 \pm 0.35$ & $4.11 \pm 0.50$ & $76.10 \pm 8.66$ \\
\hline Doxorubicin & $2.86 \pm 0.31$ & $4.16 \pm 0.40$ & $88.70 \pm 9.11$ \\
\hline
\end{tabular}

Data were expressed as Mean \pm Standard error (S.E.) of three independent experiments. N.A. is no activity.

\section{Conclusions}

The present study reports the design and synthesis of novel series of 3-(1H-pyrazol-3yl)-4H-1,2,4-triazole derivatives 7a-o as potential anticancer agents. The prepared compounds were confirmed by ${ }^{1} \mathrm{H}$ NMR, ${ }^{13} \mathrm{C}$ NMR, and HRESI-MS. The target compounds were evaluated for their anticancer activity. One-dose in-vitro anticancer test results indicated that compounds $\mathbf{7 e}$ exhibited the highest ability to inhibit the proliferation of different cancer cell lines. In-vitro five-dose full NCI 60 cell panel assay revealed that $7 \mathbf{e}$ exhibited a broad-spectrum antitumor activity against the nine tumour subpanels without pronounced selectivity. The studies confirmed that compound $\mathbf{7 e}$ is a potent lead compound for drug discovery and requires further optimization. In addition, compounds $7 \mathbf{l}$ and $7 \mathbf{o}$ were found to be highly potent and similar to doxorubicin against MCF-7 cells and A549 cells.

\section{Acknowledgment}

We are expressed great thanks to Prof. Laurent Trembleau and Prof. Marcel Jaspars School of Natural and Computing Sciences,
University of Aberdeen, UK for allowing us to do spectroscopy and HRESI-MS analysis. We are also thankful to the staff members of the National Cancer Institute (NCI), USA, for invitro anticancer screening of the newly synthesized compounds.

\section{REFERENCES}

1- WHO, Cancer, World Health Organization, February 2014, http://en.wikipedia.org/wiki/cancer.

2- G. Szabó, J. Fischer, Á. Kis-Varga and K. Gyires, "New celecoxib derivatives as anti-inflammatory agents", J. Med. Chem., 51 (1), 142-147 (2008).

3- A. P. Keche, G. D. Hatnapure, R. H. Tale, A. H. Rodge and V. M. Kamble, "Synthesis, anti-inflammatory and antimicrobial evaluation of novel 1-acetyl3,5-diaryl-4,5-dihydro (1H) pyrazole derivatives bearing urea, thiourea and sulfonamide moieties", Bioorg. Med. Chem. Lett., 22 (21), 6611-6615 (2012).

4- T. D. Penning, J. J. Talley, S. R. Bertenshaw, J. S. Carter, P. W. Collins, S. Docter, M. J. Graneto, L. F. Lee, J. W. 
Malecha, J. M. Miyashiro, R. S. Rogers, D. J. Rogier, S. S. Yu, G. D. Anderson, E. G. Burton, J. N. Cogburn, S. A. Gregory, C. M. Koboldt, W. E. Perkins, K. Seibert, A. W. Veenhuizen, Y. Y. Zhang and P. C. Isakson, "Synthesis and biological evaluation of the 1,5-diarylpyrazole class of cyclooxygenase-2 inhibitors: Identification of 4-[5-(4-methylphenyl)-3(trifluoromethyl)-1h-pyrazol-1-yl]benzene -sulfonamide (sc-58635, celecoxib)", $\boldsymbol{J}$. Med. Chem., 40 (9), 1347-1365 (1997).

5- G. Menozzi, L. Mosti, P. Fossa, F. Mattioli and M. Ghia, " $\omega$-Dialkylaminoalkyl ethers of phenyl-(5-substituted 1-phenyl-1H-pyrazol-4-yl) methanols with analgesic and anti-inflammatory activity", J. Heterocycl. Chem., 34 (3), 963-968 (1997).

6- A. Balbi, M. Anzaldi, C. Macciò, C. Aiello, M. Mazzei, R. Gangemi, P. Castagnola, M. Miele, C. Rosano and M. Viale, "Synthesis and biological evaluation of novel pyrazole derivatives with anticancer activity", Eur. J. Med. Chem., 46 (11), 5293-5309 (2011)

7- I. Vujasinović, A. Paravić-Radičević, K. Mlinarić-Majerski, K. Brajša and B. Bertoša, "Synthesis and biological validation of novel pyrazole derivatives with anticancer activity guided by 3DQSAR analysis", Bioorg. Med. Chem., 20 (6), 2101-2110 (2012).

8- D. Havrylyuk, B. Zimenkovsky, O. Vasylenko, L. Zaprutko, A. Gzella and R. Lesyk, "Synthesis of novel thiazolonebased compounds containing pyrazoline moiety and evaluation of their anticancer activity", Eur. J. Med. Chem., 44 (4), 1396-1404 (2009).

9- M. Shaharyar, M. M. Abdullah, M. A. Bakht and J. Majeed, "Pyrazoline bearing benzimidazoles: Search for anticancer agent", ibid., 45 (1), 114-119 (2010).

10- P.-C. Lv, H.-Q. Li, J. Sun, Y. Zhou, and H.-L. Zhu, "Synthesis and biological evaluation of pyrazole derivatives containing thiourea skeleton as anticancer agents", Bioorg. Med. Chem. 18 (13), 4606-4614 (2010).

11- M. S. Christodoulou, S. Liekens, K. M. Kasiotis, and S. A. Haroutounian, "Novel pyrazole derivatives: Synthesis and evaluation of anti-angiogenic activity", ibid., 18 (12), 4338-4350 (2010).

12- H.-J. Park, K. Lee, S.-J. Park, B. Ahn, J.C. Lee, H. Cho, and K.-I. Lee, "Identification of antitumor activity of pyrazole oxime ethers", Bioorg. Med. Chem. Lett., 15 (13), 3307-3312 (2005).

13- S. Manfredini, R. Bazzanini, P. G. Baraldi, M. Guarneri, D. Simoni, M. E. Marongiu, A. Pani, P. La Colla and E. Tramontano, "Pyrazole-related nucleosides. Synthesis and antiviral/antitumor activity of some substituted pyrazole and pyrazolo[4,3-d]1,2,3-triazin-4-one nucleosides", $\boldsymbol{J}$. Med. Chem., 35 (5), 917-924 (1992).

14- L.-L. Xu, C.-J. Zheng, L.-P. Sun, J. Miao and H.-R. Piao, "Synthesis of novel 1,3diaryl pyrazole derivatives bearing rhodanine-3-fatty acid moieties as potential antibacterial agents", Eur. J. Med. Chem., 48 (0), 174-178 (2012).

15- R. Sridhar, P. T. Perumal, S. Etti, G. Shanmugam, M. N. Ponnuswamy, V. R. Prabavathy and N. Mathivanan, "Design, synthesis and anti-microbial activity of 1H-pyrazole carboxylates", Bioorg. Med. Chem. Lett., 14 (24) 6035-6040 (2004).

16- M. J. Ahsan, J. G. Samy, K. R. Dutt, U. K. Agrawal, B. S. Yadav, S. Vyas, R. Kaur and G. Yadav, "Design, synthesis and antimycobacterial evaluation of novel 3substituted-N-aryl-6,7-dimethoxy-3a,4dihydro-3H-indeno[1,2-c]pyrazole-2carboxamide analogues", ibid., 21 (15), 4451-4453 (2011).

17- M. J. Ahsan, J. G. Samy, S. Soni, N. Jain, L. Kumar, L. K. Sharma, H. Yadav, L. Saini, R. G. Kalyansing, N. S. Devenda, R. Prasad and C. B. Jain, "Discovery of novel antitubercular 3a,4-dihydro-3Hindeno[1,2-c]pyrazole-2-carboxamide /carbothioamide analogues", ibid., 21 (18), 5259-5261(2011).

18- K. L. Kees, J. J. Fitzgerald, K. E. Steiner, J. F. Mattes, B. Mihan, T. Tosi, D. Mondoro and M. L. McCaleb, "New potent antihyperglycemic agents in $\mathrm{db} / \mathrm{db}$ mice: Synthesis and structure-activity relationship studies of (4-substituted benzyl)(trifluoromethyl)pyrazoles and pyrazolones", Bioorg. Med. Chem., 39 (20), 3920-3928 (1996). 
19- Y. Rajendra Prasad, A. Lakshmana Rao, L. Prasoona, K. Murali and P. Ravi Kumar, "Synthesis and antidepressant activity of some 1,3,5-triphenyl-2pyrazolines and 3-(2"-hydroxy naphthalen-1"-yl)-1,5-diphenyl-2pyrazolines", Bioorg. Med. Chem. Lett. 15 (22), 5030-5034 (2005).

20- Z. Özdemir, H. B. Kandilci, B. Gümüşel, Ü. Çalış and A. A. Bilgin, "Synthesis and studies on antidepressant and anticonvulsant activities of some 3-(2furyl)-pyrazoline derivatives", Eur. J. Med. Chem., 42 (3), 373-379 (2007).

21- M. J. Ahsan, H. Khalilullah, J. P. Stables and J. Govindasamy, "Synthesis and anticonvulsant activity of 3a,4-dihydro3H-indeno[1,2-c]pyrazole-2-carboxamide/ carbothioamide analogues", J. Enzym. Inhib. Med. Chem., 28 (3) 644-650 (2012).

22- H. Khalilullah, S. Khan, M. J. Ahsan and B. Ahmed, "Synthesis and antihepatotoxic activity of 5-(2,3-dihydro-1,4-benzodioxane-6-yl)-3-substituted-phenyl-4,5dihydro-1H-pyrazole derivatives", Bioorg. Med. Chem. Lett., 21 (24), 7251-7254 (2011).

23- H. Hashimoto, K. Imamura, J.-I. Haruta and K. Wakitani, "4-(4-Cycloalkyl/aryloxazol-5-yl)benzenesulfonamides as selective cyclooxygenase-2 inhibitors: Enhancement of the selectivity by introduction of a fluorine atom and identification of a potent, highly selective, and orally active COX-2 inhibitor JTE5221", J. Med. Chem., 45 (7), 1511-1517 (2002).

24- M. H. Ismail, J. Lehmann, D. Abou El Ella, A. Albohy and K. M. Abouzid, "Lonazolac analogues: Molecular modeling, synthesis, and in-vivo antiinflammatory activity", Med. Chem. Res., 18 (9), 725-744 (2009).

25- N. K. Terrett, A. S. Bell, D. Brown and P. Ellis, "Sildenafil (VIAGRATM), a potent and selective inhibitor of type 5 cGMP phosphodiesterase with utility for the treatment of male erectile dysfunction", Bioorg. Med. Chem. Lett., 6 (15), 18191824 (1996).

26- R. J. Nevagi, "Recent advances in bioactive pyrazole scaffold-Part III: Anti- cancer agents", Der Pharmacia Lettre, 6 (5), 285-295 (2014).

27- B. Costa, "Rimonabant: More than an antiobesity drug?", Br. J. Pharmacol., 150 (5), 535-537 (2007).

28- R. G. Pertwee, "Pharmacology of cannabinoid receptor ligands", Curr. Med. Chem., 6 (8), 635-664 (1999).

29- C. Blazquez, M. L. Casanova, A. Planas, T. G. Del Pulgar, C. Villanueva, M. J. Fernandez-Acenero, J. Aragones, J. W. Huffman, J. L. Jorcano and M. Guzman, "Inhibition of tumor angiogenesis by cannabinoids", The FASEB Journal: Official Publication of the Federation of American Societies for Experimental Biology, 17 (3), 529-531 (2003).

30- A. Hasan, N. F. Thomas and S. Gapil, "Synthesis, characterization and antifungal evaluation of 5-substituted-4-amino-1,2,4triazole-3-thioesters", Molecules, 16 (2), 1297-1309 (2011).

31- H. Bayrak, A. Demirbas, S. A. Karaoglu and N. Demirbas, "Synthesis of some new 1,2,4-triazoles, their Mannich and Schiff bases and evaluation of their antimicrobial activities", Eur. J. Med. Chem., 44 (3), 1057-1066 (2009).

32- I. Küçükgüzel, S. G. Küçükgüzel, S. Rollas and M. Kiraz, "Some 3thioxo/alkylthio-1,2,4-triazoles with a substituted thiourea moiety as possible antimycobacterials", Bioorg. Med. Chem. Lett., 11 (13), 1703-1707 (2001).

33- B. Shivarama Holla, K. Narayana Poojary, B. Sooryanarayana Rao and M. K. Shivananda, "New bis-aminomercaptotriazoles and bis-triazolothiadiazoles as possible anticancer agents", Eur. J. Med. Chem., 37 (6), 511-517 (2002).

34- B. Shivarama Holla, B. Veerendra, M. K. Shivananda and B. Poojary, "Synthesis characterization and anticancer activity studies on some Mannich bases derived from 1,2,4-triazoles", ibid., 38 (7-8), 759767 (2003).

35- K. Sztanke, T. Tuzimski, J. Rzymowska, K. Pasternak and M. Kandefer-Szerszeń, "Synthesis, determination of the lipophilicity, anticancer and antimicrobial properties of some fused 1,2,4-triazole derivatives", ibid., 43 (2), 404-419 (2008). 
36- K. S. Bhat, B. Poojary, D. J. Prasad, P. Naik and B. S. Holla, "Synthesis and antitumor activity studies of some new fused 1,2,4-triazole derivatives carrying 2,4-dichloro-5-fluorophenyl moiety", ibid., 44 (12), 5066-5070 (2009).

37- Y. A. Al-Soud, M. N. Al-Dweri and N. A. Al-Masoudi, "Synthesis, antitumor and antiviral properties of some 1,2,4-triazole derivatives", Il Farmaco., 59 (10), 775783 (2004).

38- N. Demirbaş, R. Ugurluoglu and A. Demirbaş, "Synthesis of 3-alkyl(Aryl)-4alkylidenamino-4,5-dihydro-1H-1,2,4triazol-5-ones and 3-alkyl-4-alkylamino4,5-dihydro-1H-1,2,4-triazol-5-ones as antitumor agents", Bioorg. Med. Chem., 10 (12), 3717-3723 (2002).

39- M. Kritsanida, A. Mouroutsou, P. Marakos, N. Pouli, S. PapakonstantinouGaroufalias, C. Pannecouque, M. Witvrouw and E. De Clercq, "Synthesis and antiviral activity evaluation of some new 6-substituted 3-(1-adamantyl)-1,2,4triazolo[3,4-b][1,3,4]thiadiazoles", Il Farmaco., 57 (3), 253-257 (2002).

40- A. Almasirad, S. A. Tabatabai, M. Faizi, A. Kebriaeezadeh, N. Mehrabi, A. Dalvandi and A. Shafiee, "Synthesis and anticonvulsant activity of new 2substituted-5-[2-(2-fluorophenoxy)phenyl] -1,3,4-oxadiazoles and 1,2,4-triazoles", Bioorg. Med. Chem. Lett., 14 (24), 60576059 (2004).

41- G. Turan-Zitouni, Z. A. Kaplancikli, A. Özdemir, P. Chevallet, H. B. Kandilci and B. Gümüsel, "Studies on 1,2,4-triazole derivatives as potential anti-inflam-matory agents", Arch. Pharm. 340 (11), 586-590 (2007).

42- G. Turan-Zitouni, Z. A. Kaplancikli, K. Erol and F. S. Kiliç, "Synthesis and analgesic activity of some triazoles and triazolothiadiazines", Il Farmaco., 54 (4), 218-223 (1999).

43- H. Emilsson, H. Selander and J. Gaarder, "Synthesis and antihypertensive activity of some 3-substituted 4H-1,2,4-triazoles", Acta Pharm. Suec., 24, 123-134 (1987).

44- C. Radhika, A. Venkatesham and M. Sarangapani, "Synthesis and antidepressant activity of di substituted-5- aryl-1,2,4-triazoles", Med. Chem. Res., 21 (11), 3509-3513 (2012). 
45- S. C. Holm and B. F. Straub, "Synthesis of n-substituted 1,2,4-triazoles. A review", Org. Prep. Proced. Int., 43 (4), 319-347 (2011).

46- The methodology of the NCI anticancer screening has been described in detail elsewhere. http://www.dtp.nci.nih.gov.

47- A. Monks, D. Scudiero, P. Skehan, R. Shoemaker, K. Paull, D. Vistica, C. Hose, J. Langley, P. Cronise, A. Vaigro-Wolff, M. Gray-Goodrich, H. Campbell, J. Mayo and M. Boyd, "Feasibility of a high-flux anticancer drug screen using a diverse panel of cultured human tumor cell lines", J. Natl. Cancer Inst., 83 (11), 757-766 (1991).

48- R. H. Shoemaker, "The NCI60 human tumour cell line anticancer drug screen", Nat. Rev. Cancer, 6 (10), 813-823 (2006).

49- P. Skehan, R. Storeng, D. Scudiero, A. Monks, J. McMahon, D. Vistica, J. T. Warren, H. Bokesch, S. Kenney and M. R. Boyd, "New colorimetric cytotoxicity assay for anticancer-drug screening", $\boldsymbol{J}$. Natl. Cancer Inst., 82, 1107-1112 (1990).

50- C. H. Wu, M. S. Hung, J. S. Song, T. K. Yeh, M. C. Chou, C. M. Chu, J. J. Jan, M. T. Hsieh, S. L. Tseng, C. P. Chang, W. P. Hsieh, Y. Lin, Y. N. Yeh, W. L. Chung, C. W. Kuo, C. Y. Lin, H. S. Shy, Y. S. Chao and K. S. Shia, "Discovery of 2-[5-(4chloro-phenyl)-1-(2,4-dichloro-phenyl)-4ethyl-1H- pyrazol-3-yl]-1,5,5-trimethyl1,5-dihydro-imidazol-4-thione (BPR-890) via an active metabolite. A novel, potent and selective cannabinoid-1 receptor inverse agonist with high antiobesity efficacy in DIO mice", J. Med. Chem., 52 (14), 4496-4510 (2009). 


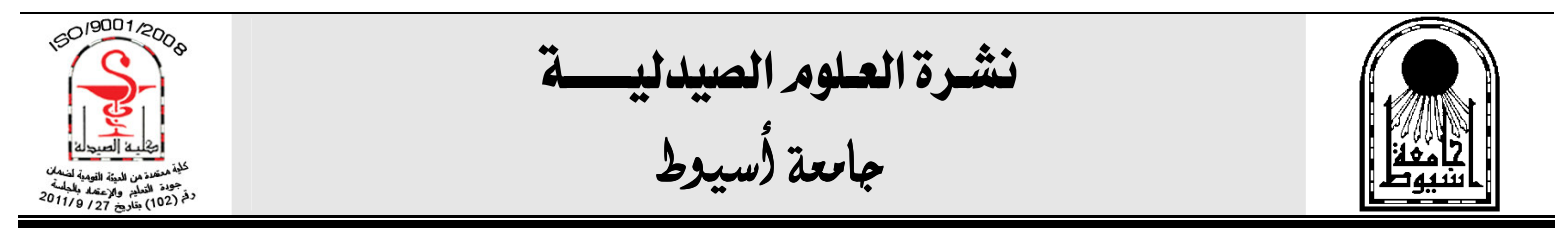

تثييد مشتقات جديدة من البيرازول حاملات I،r، ع-تريازول

كمضادات محتملة للسرطان

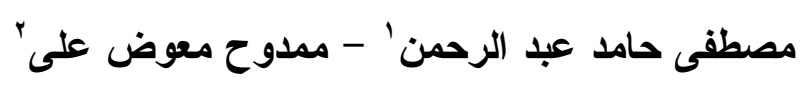

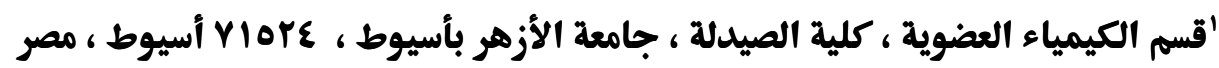

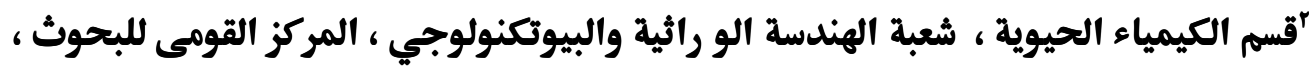

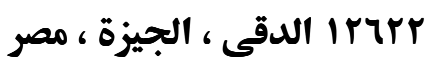

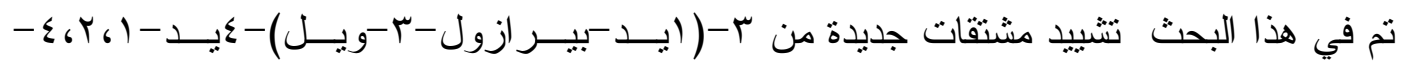

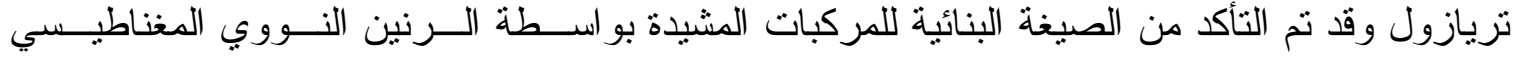

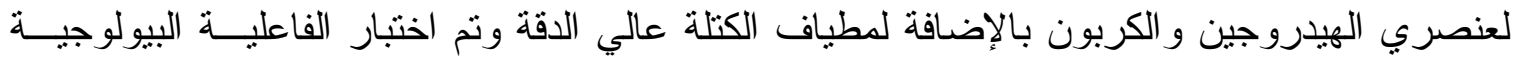

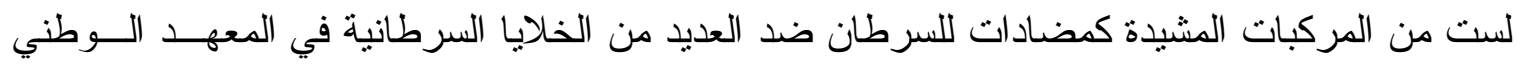

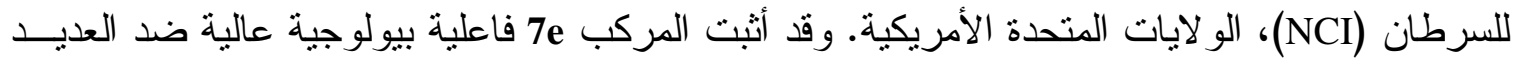

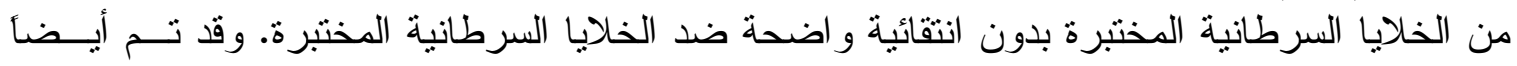

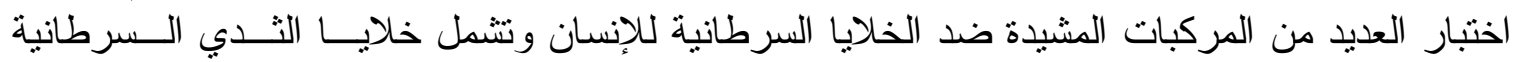

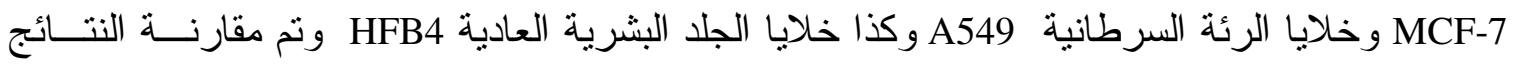

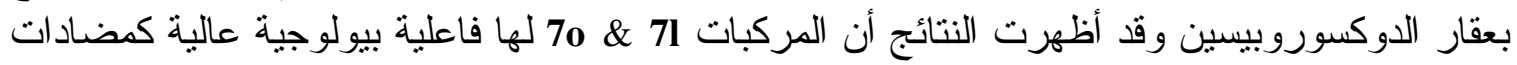

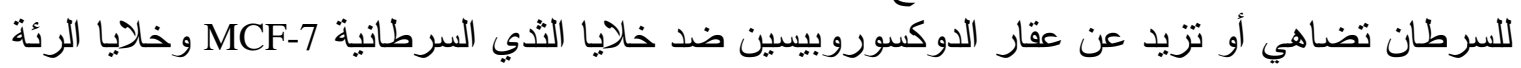

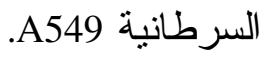

